\title{
ASAM v2.7: a compressible atmospheric model with a Cartesian cut cell approach
}

\author{
M. Jähn, O. Knoth, M. König, and U. Vogelsberg \\ Leibniz Institute for Tropospheric Research, Permoserstrasse 15, 04318 Leipzig, Germany \\ Correspondence to: M. Jähn (jaehn@tropos.de)
}

Received: 16 June 2014 - Published in Geosci. Model Dev. Discuss.: 18 July 2014

Revised: 19 January 2015 - Accepted: 22 January 2015 - Published: 18 February 2015

\begin{abstract}
In this work, the fully compressible, threedimensional, nonhydrostatic atmospheric model called All Scale Atmospheric Model (ASAM) is presented. A cut cell approach is used to include obstacles and orography into the Cartesian grid. Discretization is realized by a mixture of finite differences and finite volumes and a state limiting is applied. Necessary shifting and interpolation techniques are outlined. The method can be generalized to any other orthogonal grids, e.g., a lat-long grid. A linear implicit Rosenbrock time integration scheme ensures numerical stability in the presence of fast sound waves and around small cells. Analyses of five two-dimensional benchmark test cases from the literature are carried out to show that the described method produces meaningful results with respect to conservation properties and model accuracy. The test cases are partly modified in a way that the flow field or scalars interact with cut cells. To make the model applicable for atmospheric problems, physical parameterizations like a Smagorinsky subgrid-scale model, a two-moment bulk microphysics scheme, and precipitation and surface fluxes using a sophisticated multi-layer soil model are implemented and described. Results of an idealized three-dimensional simulation are shown, where the flow field around an idealized mountain with subsequent gravity wave generation, latent heat release, orographic clouds and precipitation are modeled.
\end{abstract}

\section{Introduction}

In this paper we present the numerical solver ASAM (All Scale Atmospheric Model) that has been developed at the Leibniz Institute for Tropospheric Research (TROPOS),
Leipzig. ASAM was initially designed for CFD (computational fluid dynamics) simulations around buildings. The model can also be used with spherical or cylindrical grids. Stability problems with grid convergence in special points (the pole problem) in both grids are handled through the implicit time integration both for advection and the yet faster gravity and acoustic waves. For simulating the flow around obstacles, buildings or orography, the cut (or shaved) cell approach is used. With this attempt one remains within the Cartesian grid and the numerical pressure derivative in the vicinity of a structure is zero if the cut cell geometry is not taken into account, which is not the case in terrain-following coordinate systems due to the slope of the lowest cells (Lock et al., 2012). Since this skewness is also reproduced in upper levels, a cut cell model produces reduced or greatly reduced errors in comparison models with terrain-following coordinates (Good et al., 2014). Several techniques have been developed to overcome these nonphysical errors associated with terrain-following grids, especially when spatial scales of three-dimensional models become finer (which leads to a steepening of the model orography). Tripoli and Smith (2014a) introduced a variable-step topography (VST) surface coordinate system within a nonhydrostatic host model. Unlike the traditional discrete-step approach, the depth of a grid box intersecting with a topographical structure is adjusted to its height, which leads to straight cut cells. $\mathrm{Nu}$ merical tests show that this technique produces better results than conventional approaches for different topography (severe and smooth) types (Tripoli and Smith, 2014b). In their cases, also the computational costs with the VST approach are reduced because there is no need of extra functional transform calculations due to metric terms. Steppeler et al. (2002) derived approximations for $z$ coordinate non- 
hydrostatic atmospheric models by using the shaved-element finite volume method. There, the dynamics are computed in the cut cell system, whereas the physics computation remains in the terrain-following system. Using a $z$-coordinate system can also improve the prediction of meteorological parameters like clouds and rainfall due to a better representation of the atmospheric flow near mountains in a numerical weather prediction (NWP) model (Steppeler et al., 2006, 2013). The cut cell method is also used in the Ocean-Land-Atmosphere Model (OLAM) (Walko and Avissar, 2008a), which extends the Regional Atmospheric Modeling System (RAMS) to a global model domain. In OLAM, the shaved-cell method is applied to an icosahedral mesh (Walko and Avissar, 2008b). Yamazaki and Satomura (2008) simulated a two-dimensional flow over different mountain slopes and compared the results of their cut cell model with a model using terrain-following coordinates. Especially for steep slopes, significant errors were reported in the terrain-following model. A drawback is the generation of low-volume cells when a cut cell method is used. To avoid instability problems around these small cells, the time integration scheme has to be adapted. This can be achieved by using semi-implicit or semi-Langrangian methods, for example. In ASAM, a linear-implicit Rosenbrock time integration scheme is used (Hairer and Wanner, 1996). Another option to handle the small cells problem is to merge small cut cells with neighboring cells in either the horizontal or vertical direction (Yamazaki and Satomura, 2010). However, this approach becomes more complicated when applying it to three spatial dimensions, since a lot of special cases have to be considered. To achieve reasonable vertical solutions near the ground, the usage of local mesh refinement techniques becomes interesting for large-scale models (Yamazaki and Satomura, 2012).

The here-presented model is a developing research code with different options to choose from like different numerical methods (e.g., split-explicit Runge-Kutta or partially implicit peer schemes), number of prognostic variables, physical parameterizations or the change to a spherical grid type. Parallelization is realized by using the message passing interface (MPI) and the domain decomposition method. The code is easily portable between different platforms like Linux, IBM or Mac OS. With these features, large eddy simulations (LES) with spatial resolutions of $\mathcal{O}(1-100 \mathrm{~m})$ can be performed with respect to a sufficiently resolved terrain structure. In previous studies, the model was used to demonstrate the volume-of-fluid (VOF) method for nondissipative cloud transport (Hinneburg and Knoth, 2005). ASAM also took part in an intercomparison study of mountain-wave simulations for idealized and real terrain profiles, where altogether 11 different nonhydrostatic numerical models were compared (Doyle et al., 2011). A partially implicit peer method is presented in Jebens et al. (2011) in order to overcome the small cell problem around orography when using cut cells. The model was recently used for a study of dynamic flow structures in a turbulent urban environment of a building- resolving resolution (König, 2013). There, the implementation of a dynamic Smagorinsky subgrid-scale model is tested for a convective atmospheric boundary layer and an inflow generation approach that produces a turbulent flow field is presented.

A separately developed LES model at TROPOS is called ASAMgpu (Horn, 2012). It includes some basic features of the ASAM code and runs on graphics processing units (GPUs), which enables very time-efficient computations and post-processing. However, this model is not as adjustable as the original ASAM code and the inclusion of threedimensional orographical structures is not implemented so far. ASAMgpu was applied for a study of heat island effects on vertical mixing of aerosols by comparing the results of large eddy simulations with wind and aerosol lidar observations (Engelmann et al., 2011).

This paper is structured as follows. The next section deals with a general description of the model. It includes the basic equations that are solved numerically and the used energy variable. Also, the cut cell approach and spatial discretization as well as the time integration scheme are described. This approach can be extended to other orthogonal grids like the latlong grid. Section 3 deals with the model physics, including a subgrid-scale model, a two-moment microphysics scheme and surface flux parameterization. Results of different idealized test cases are shown in Sect. 4. The first one is a dry rising heat bubble described by Wicker and Skamarock (1998) followed by a flow past an idealized mountain ridge from Schaer et al. (2002). Two other "classical" test examples have been chosen and modified so that cut cells are included and interaction within these cells are guaranteed. The first one is a falling cold bubble with a developing density current (Straka et al., 1993) but with a $1 \mathrm{~km}$ high mountain on the left part of the domain. The second case is the moist bubble benchmark case reported by Bryan and Fritsch (2002). The bubble will rise and interact with a zeppelin-shaped cut area in the center of the domain. For both test cases, energy conservation tests are carried out. Another two-dimensional case by Berger and Helzel (2012) is presented to test the accuracy of the cut cell method by advecting a smooth bump in a radial wind field in an annulus. For the last test case, a three-dimensional mountain overflow with subsequent orographic cloud generation and precipitation is simulated (Kunz and Wassermann, 2011). Concluding remarks and future work are in the final section.

\section{Description of the All Scale Atmospheric Model}

\subsection{Governing equations}

The flux-form compressible Euler equations for the atmosphere are 
Table 1. Physical constants.

\begin{tabular}{cll}
\hline Symbol & Quantity & Value \\
\hline$p_{0}$ & Reference pressure & $10^{5} \mathrm{~Pa}$ \\
$R_{\mathrm{d}}$ & Gas constant for dry air & $287 \mathrm{~J} \mathrm{~kg}^{-1} \mathrm{~K}^{-1}$ \\
$R_{\mathrm{V}}$ & Gas constant for water vapor & $461 \mathrm{~J} \mathrm{~kg}^{-1} \mathrm{~K}^{-1}$ \\
$c_{\mathrm{pd}}$ & Specific heat capacity at constant pressure for dry air & $1004 \mathrm{~J} \mathrm{~kg}^{-1} \mathrm{~K}^{-1}$ \\
$c_{\mathrm{pv}}$ & Specific heat capacity at constant pressure for water vapor & $1885 \mathrm{~J} \mathrm{gg}^{-1} \mathrm{~K}^{-1}$ \\
$c_{\mathrm{pl}}$ & Specific heat capacity at constant pressure for liquid water & $4186 \mathrm{~J} \mathrm{~kg}^{-1} \mathrm{~K}^{-1}$ \\
$c_{\mathrm{vd}}$ & Specific heat capacity at constant volume for dry air & $717 \mathrm{~J} \mathrm{gg}^{-1} \mathrm{~K}^{-1}$ \\
$c_{\mathrm{vv}}$ & Specific heat capacity at constant volume for water vapor & $1424 \mathrm{~J} \mathrm{~kg}^{-1} \mathrm{~K}^{-1}$ \\
$L_{00}$ & Latent heat at $0 \mathrm{~K}$ & $3.148 \times 10^{6} \mathrm{~J} \mathrm{~kg}^{-1}$ \\
$g$ & Gravitational acceleration & $9.81 \mathrm{~m} \mathrm{~s}^{-2}$ \\
$C_{\mathrm{S}}$ & Smagorinsky coefficient & 0.2 \\
\hline
\end{tabular}

\subsection{Cut cells and spatial discretization}

$$
\begin{aligned}
\frac{\partial \rho}{\partial t}+\nabla \cdot(\rho \boldsymbol{v}) & =0, \\
\frac{\partial(\rho \boldsymbol{v})}{\partial t}+\nabla \cdot(\rho \boldsymbol{v} \boldsymbol{v}) & =-\nabla \cdot \boldsymbol{\tau}-\nabla p-\rho \boldsymbol{g}-2 \Omega \times(\rho \boldsymbol{v}), \\
\frac{\partial(\rho \phi)}{\partial t}+\nabla \cdot(\rho \boldsymbol{v} \phi) & =-\nabla \cdot \boldsymbol{q}_{\phi}+S_{\phi},
\end{aligned}
$$

where $\rho$ is the total air density, $v=(u, v, w)^{\mathrm{T}}$ the threedimensional velocity vector, $p$ the air pressure, $g$ the gravitational acceleration, $\Omega$ the angular velocity vector of the earth, $\phi$ a scalar quantity and $S_{\phi}$ the sum of its corresponding source terms. The subgrid-scale terms are $\boldsymbol{\tau}$ for momentum and $\boldsymbol{q}_{\phi}$ for a given scalar.

The energy equation in the form of Eq. (3) is represented by the (dry) potential temperature $\theta$. In the presence of water vapor and cloud water, this quantity is replaced by the density potential temperature $\theta_{\rho}$ (Emanuel, 1994) as a more generalized form of the virtual potential temperature $\theta_{\mathrm{v}}$ :

$\theta_{\rho}=\theta\left(1+q_{\mathrm{v}}\left[\frac{R_{\mathrm{v}}}{R_{\mathrm{d}}}-1\right]-q_{\mathrm{c}}\right)$,

where the equation of state can be expressed as follows:

$p=\rho R_{\mathrm{d}} \theta_{\rho}\left(\frac{p}{p_{0}}\right)^{\kappa_{\mathrm{m}}}$.

In the previous two equations $\theta=T\left(p_{0} / p\right)^{\kappa_{\mathrm{m}}}$ is the potential temperature, $q_{\mathrm{v}}=\rho_{\mathrm{v}} / \rho$ is the mass ratio of water vapor in the air (specific humidity), $q_{\mathrm{c}}=\rho_{\mathrm{c}} / \rho$ is the mass ratio of cloud water in the air, $p_{0}$ a reference pressure and $\kappa_{\mathrm{m}}=\left(q_{\mathrm{d}} R_{\mathrm{d}}+\right.$ $\left.q_{\mathrm{v}} R_{\mathrm{v}}\right) /\left(q_{\mathrm{d}} c_{\mathrm{pd}}+q_{\mathrm{v}} c_{\mathrm{pv}}+q_{\mathrm{c}} c_{\mathrm{pl}}\right)$ the Poisson constant for the air mixture (dry air, water vapor, cloud water) with $q_{\mathrm{d}}=\rho_{\mathrm{d}} / \rho$. $R_{\mathrm{d}}$ and $R_{\mathrm{V}}$ are the gas constants for dry air and water vapor, respectively.

The number of additional equations like Eq. (3) depends on the complexity of the microphysical scheme used. Furthermore, tracer variables can also be included. The values of all relevant physical constants are listed in Table 1.

\subsubsection{Definition of cut cells}

The spatial discretization is done on a Cartesian grid with grid intervals of lengths $\Delta x_{i}, \Delta y_{j}, \Delta z_{k}$ and can easily be extended to any orthogonal, logically rectangular structured grid (i.e., it has the same logical structure as a regular Cartesian grid) like spherical or cylindrical coordinates. First, it is described for the Cartesian case. Generalizations are discussed afterwards. Orography and other obstacles like buildings are presented by cut cells, which are the result of the intersection of the obstacle with the underlying Cartesian grid. In Fig. 1 different possible and excluded configurations are shown for the three-dimensional case. For the spatial discretization only the six partial face areas and the partial cell volume and the grid sizes of the underlying Cartesian mesh are used. For a proper representation the orography is smoothed in such a way that the intersection of a grid cell and the orography can be described by a single possible nonplanar polygon. Or in other words, a Cartesian cell is divided in at most two parts, a free part and a solid part. For each Cartesian cell, the free face area of the six faces and the free volume area of the cell are stored, which is the part outside of the obstacle. These values are denoted for the grid cell $i, j, k$ by $\mathrm{FU}_{i-1 / 2, j, k}, \mathrm{FU}_{i+1 / 2, j, k}, \mathrm{FV}_{i, j-1 / 2, k}, \mathrm{FV}_{i, j+1 / 2, k}$, $\mathrm{FW}_{i, j, k-1 / 2}, \mathrm{FW}_{i, j, k+1 / 2}$ and $\mathrm{V}_{i, j, k}$, respectively. In the following, the relative notations $\mathrm{FU}_{\mathrm{L}}$ and $\mathrm{FU}_{\mathrm{R}}$ are used, e.g., as shown in Fig. 3.

\subsubsection{Spatial discretization}

The spatial discretization is formulated in terms of the grid interval length and the face and volume areas. The variables are arranged on a staggered grid with momentum $\mathbf{V}=(U, V, W)=(\rho u, \rho v, \rho w)$ at the cell faces and all other variables at the cell center. The discretization is a mixture of finite volumes and finite differences. In the finite volume 


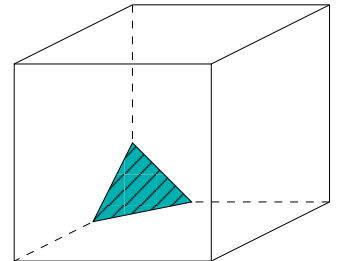

3 markers

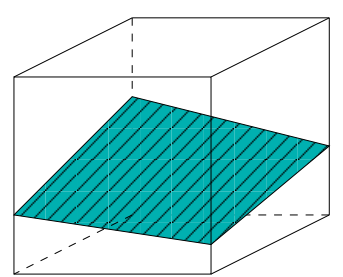

4 markers

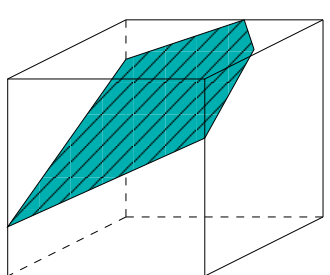

5 markers

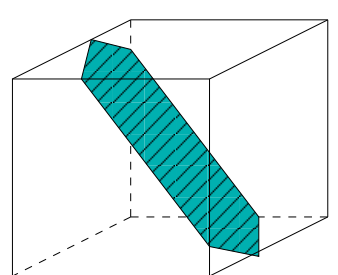

6 markers

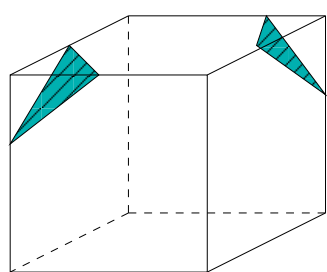

6 markers

Figure 1. Possible configurations for cut cell intersection (cases 1-3) for different numbers of face intersection points (markers). The last two cases are excluded.

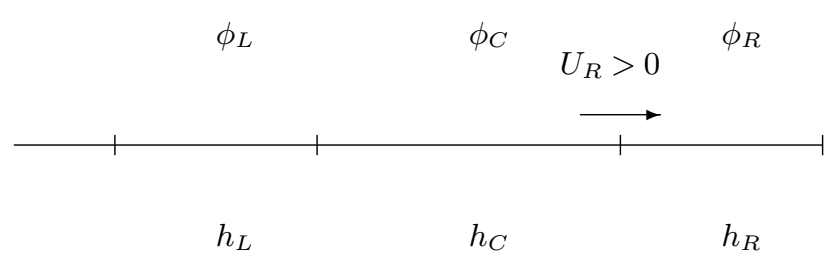

Figure 2. Stencil for third-order approximation.
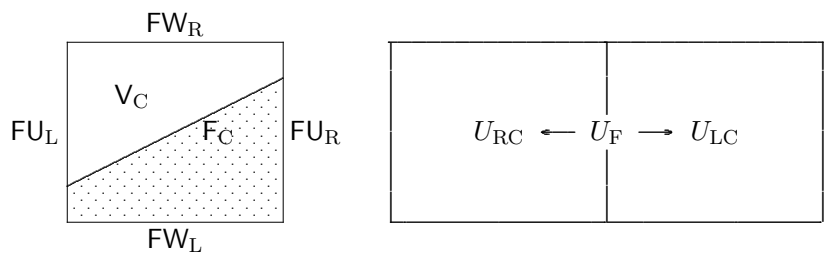

Figure 3. Cut cell with face and volume area information (left) and arrangement of face- and cell-centered momentum (right).

context the main task is the reconstruction of values and gradients at cell faces from cell-centered values.

The discretization of the advection operator is performed for a generic cell-centered scalar variable $\phi$. In the context of a finite volume discretization, point values of the scalar value $\phi$ are needed at the faces of this grid cell. Knowing these face values, the advection operator in the $x$ direction is discretized by $\left(\mathrm{FU}_{\mathrm{R}} U_{\mathrm{FR}} \phi_{\mathrm{R}}-\mathrm{FU}_{\mathrm{L}} U_{\mathrm{FL}} \phi_{\mathrm{L}}\right) / \mathrm{V}_{\mathrm{C}}$ where $U_{\mathrm{F}}$ is the discretized momentum at the corresponding faces. To approximate these values at the faces, a biased upwind thirdorder procedure with additional limiting is used (Van Leer, 1994).

Assuming a positive flow in the $x$ direction, the third-order approximation at $x_{i+1 / 2}$ is obtained by quadratic interpolation from the three values as shown in Fig. 2. The interpolation condition is that the three cell-averaged values are fitted:

$$
\begin{aligned}
\phi_{\mathrm{FR}}= & \phi_{\mathrm{C}}+\frac{h_{\mathrm{C}}\left(h_{\mathrm{L}}+h_{\mathrm{C}}\right)}{\left(h_{\mathrm{C}}+h_{\mathrm{R}}\right)\left(h_{\mathrm{L}}+h_{\mathrm{C}}+h_{\mathrm{R}}\right)}\left(\phi_{\mathrm{R}}-\phi_{\mathrm{C}}\right) \\
& +\frac{h_{\mathrm{C}} h_{\mathrm{R}}}{\left(h_{\mathrm{L}}+h_{\mathrm{C}}\right)\left(h_{\mathrm{L}}+h_{\mathrm{C}}+h_{\mathrm{R}}\right)}\left(\phi_{\mathrm{C}}-\phi_{\mathrm{L}}\right) \\
= & \phi_{\mathrm{C}}+\alpha_{1}\left(\phi_{\mathrm{R}}-\phi_{\mathrm{C}}\right)+\alpha_{2}\left(\phi_{\mathrm{C}}-\phi_{\mathrm{L}}\right) .
\end{aligned}
$$

To achieve positivity in Eq. (6), we apply state limiting. For this task, Eq. (6) is rewritten in slope-ratio formulation:

$\phi_{\mathrm{FR}}=\phi_{\mathrm{C}}+K\left(\phi_{\mathrm{C}}-\phi_{\mathrm{L}}\right)$,

where

$K=\alpha_{1} \frac{\phi_{\mathrm{R}}-\phi_{\mathrm{C}}}{\phi_{\mathrm{C}}-\phi_{\mathrm{L}}}+\alpha_{2}$.

Then $K$ is replaced by limiter function $\Psi$ and Eq. (7) is rewritten as

$\phi_{\mathrm{FR}}=\phi_{\mathrm{C}}+\Psi\left(\frac{\phi_{\mathrm{R}}-\phi_{\mathrm{C}}}{\phi_{\mathrm{C}}-\phi_{\mathrm{L}}}\right)\left(\phi_{\mathrm{C}}-\phi_{\mathrm{L}}\right)$,

$\Psi(r)=\max \left(0, \min \left[r, \min \left(\delta, \alpha_{1} r+\alpha_{2}\right)\right]\right), \quad \delta=2$,

as proposed by Sweby (1984). This limiter has the property that the unlimited higher-order scheme (Eq. 6) is used as much as possible and is utilized only then when it is needed. In the case of $\Psi=0$, the scheme degenerates to the simple first-order upwind scheme. The coefficients $\alpha_{1}$ and $\alpha_{2}$ can be computed in advance to minimize the overhead for a nonuniform grid. In the case of a uniform grid the coefficients are constant, i.e., they are equal to $1 / 3$ and $1 / 6$. For a detailed discussion of the benefits of this approach and numerical experiments also see Hundsdorfer et al. (1995). This procedure is applied in all three grid directions, where the virtual grid sizes $h$ are defined as

$h_{\mathrm{L}}=\mathrm{V}_{\mathrm{L}} / \mathrm{F}_{\mathrm{L}}$,

$h_{\mathrm{C}}=0.5 \mathrm{~V}_{\mathrm{C}} /\left(\mathrm{F}_{\mathrm{L}}+\mathrm{F}_{\mathrm{R}}\right)$,

$h_{\mathrm{R}}=\mathrm{V}_{\mathrm{R}} / \mathrm{F}_{\mathrm{R}}$.

\subsubsection{Momentum}

To solve the momentum equation, the nonlinear advection term is needed on the face. This is achieved by a shifting technique introduced by Hicken et al. (2005) for the incompressible Navier-Stokes equation. For each cell, two cellcentered values of each of the three components of the Cartesian velocity vector are computed and transported with the above advection scheme for a cell-centered scalar value. The 
obtained tendencies are then interpolated back to the faces. This approach avoids separate advection routines for the momentum components. For a normal cell the shifted values are obtained from the six momentum face values, whereas for a cut cell the shift operation takes into account the weights of the faces of the two opposite sides, compare with Fig. 3 for the used notation.

$U_{\mathrm{LC}}= \begin{cases}U_{\mathrm{FL}} & \text { if } \mathrm{FU}_{\mathrm{L}} \geq \mathrm{FU}_{\mathrm{R}} \\ \left(U_{\mathrm{FL}} \mathrm{FU}_{\mathrm{L}}+U_{\mathrm{FR}}\left(\mathrm{FU}_{\mathrm{R}}-\mathrm{FU}_{\mathrm{L}}\right)\right) / \mathrm{FU}_{\mathrm{R}} & \text { else. }\end{cases}$

The interpolation of the cell tendencies $T U_{\mathrm{LC}}$ and $T U_{\mathrm{RC}}$ back to a face tendency $T U_{\mathrm{F}}$ is obtained by the arithmetic mean of the two tendencies of the two shifted cell components originating from the same face. For a cut face the interpolation takes the form (see Fig. 4)

$$
\begin{aligned}
T U_{\mathrm{F}}= & \left(\frac{T U_{\mathrm{RC}} \mathrm{V}_{\mathrm{L}}}{\mathrm{FU}_{\mathrm{L}}+\mathrm{FU}_{\mathrm{C}}}+\frac{T U_{\mathrm{LC}} \mathrm{V}_{\mathrm{R}}}{\mathrm{FU}_{\mathrm{R}}+\mathrm{FU}_{\mathrm{C}}}\right) / \\
& \left(\frac{\mathrm{V}_{\mathrm{L}}}{\mathrm{FU}_{\mathrm{L}}+\mathrm{FU}_{\mathrm{C}}}+\frac{\mathrm{V}_{\mathrm{R}}}{\mathrm{FU}_{\mathrm{R}}+\mathrm{FU}_{\mathrm{C}}}\right) .
\end{aligned}
$$

The pressure gradient and the Buoyancy term are computed for all faces with standard difference and interpolation formulas with the grid sizes taken from the underlying Cartesian grid. To approximate the pressure gradient at the interface of two grid cells with only the pressure values of the two grid cells there is some freedom in choosing the grid size. Whereas in Adcroft et al. (1997) the grid size is chosen to preserve energy in their model, we follow $\mathrm{Ng}$ et al. (2009) and do not take in to account the cut cell structure. Both versions are implemented in the ASAM code and it became apparent that the second one is more suitable to simulate flows in hydrostatic balance.

\subsubsection{Boundary flux distribution near cut cells}

Due to small cell volumes around cut cells, boundary fluxes such as sensible and latent surface heat fluxes (cf. Sect. 3.4) have to be distributed to the surrounding cells to avoid instability problems because of sharp gradients in the respective scalar fields. For simplicity we consider a two-dimensional case in $x$ and $z$ directions. An example configuration is displayed in Fig. 5. The common partial face area of two neighboring cells is greater than the opposite face (i.e., left vs. right in $x$ and $y$ directions, bottom vs. top), e.g., in our case $\mathrm{FU}_{\mathrm{L}}>\mathrm{FU}_{\mathrm{R}}$ and $\mathrm{FW}_{\mathrm{T}}>\mathrm{FW}_{\mathrm{B}}$. For the flux distribution we first define a virtual volume over the cut cell face $\mathrm{F}_{\mathrm{C}}$ through

$V_{\text {virt }}=\mathrm{F}_{\mathrm{C}} h_{\mathrm{virt}}$,

where

$h_{\mathrm{virt}}=\Delta x\left|n_{x}\right|+\Delta z\left|n_{z}\right|$.

Here, $\left(n_{x}, n_{z}\right)^{\mathrm{T}}$ is the normal unit vector of the cut cell face $\mathrm{F}_{\mathrm{C}}$. Then the flux fraction with a weight of $\mathrm{V}_{\mathrm{C}} / V_{\text {virt }}$

\begin{tabular}{|c|c|c|}
\hline \multicolumn{3}{|l|}{0} \\
\hline $2 / 3$ & & \\
\hline $\begin{array}{lr}-5 / 4 & 3 / 4\end{array}$ & $-4 / 3$ & $\frac{1}{2}+\frac{1}{6} \sqrt{3}$ \\
\hline $\mathcal{A}$-Matrix & $\Gamma$-Matrix & $\gamma$ \\
\hline
\end{tabular}

Table 2. Coefficient table for ROS2.

Table 3. Coefficient table for ROSRK3.

\begin{tabular}{ll|cc|c}
\hline 0 & 0 & & \\
$1 / 3$ & $-11 / 27$ & \\
$11 / 54 \quad 1 / 2$ & $17 / 27$ & $-11 / 4$ & \\
\hline $\begin{array}{c}-17 / 27 \quad 11 / 4 \\
\mathcal{A} \text {-Matrix }\end{array}$ & 1 & $\Gamma$-Matrix & $\gamma$ \\
\hline
\end{tabular}

is added to the cut cell (cell 2 in Fig. 5). The remaining part that has to be distributed is weighted by the face values $\left|\mathrm{FU}_{\mathrm{L}}-\mathrm{FU}_{\mathrm{R}}\right| / \mathrm{F}_{\text {surf }} \cdot \mathrm{V}_{\mathrm{C}} /(\Delta x \Delta y \Delta z)$ to the neighbored cells in $x$ direction (cell 1 in the example) and $\mid \mathrm{FW}_{\mathrm{B}}-$ $\mathrm{FW}_{\mathrm{T}} \mid / \mathrm{F}_{\text {surf }} \cdot \mathrm{V}_{\mathrm{C}} /(\Delta x \Delta y \Delta z)$ in $z$ direction (cell 4 in the example), where $\mathrm{F}_{\text {surf }}=\left|\mathrm{FU}_{\mathrm{L}}-\mathrm{FU}_{\mathrm{R}}\right|+\left|\mathrm{FW}_{\mathrm{B}}-\mathrm{FW}_{\mathrm{T}}\right|$. With this approach, only the available information of the considered cut cell (volume and common face areas with neighboring cells) and not of its surrounding cells is needed. The extension of this method to the third spatial dimension is done analogously.

\subsection{Time integration}

After spatial discretization, an ordinary differential equation

$y^{\prime}(t)=F(y(t))$

is obtained that has to be integrated in time (method of lines). To tackle the small time step problem connected with tiny cut cells, linear implicit Rosenbrock W methods are used (Knoth, 2006; Jebens et al., 2011; John and Rang, 2010).

A Rosenbrock method has the form

$$
\begin{aligned}
& (\mathbf{I}-\tau \gamma \mathbf{J}) k_{i}=\tau F\left(y_{n}+\sum_{j=1}^{i-1} \alpha_{i j} u_{j}\right)+\sum_{j=1}^{i-1} \gamma_{i j} k_{j}, \\
& i=1, \ldots, s, \\
& y_{n+1}=y_{n}+\sum_{j=1}^{s} \alpha_{s+1 j} k_{j},
\end{aligned}
$$

where $y_{n}$ is a given approximation at $y(t)$ at time $t_{n}$ and subsequently $y_{n+1}$ at time $t_{n+1}=t_{n}+\tau$. In addition, $\mathbf{J}$ is an approximation to the Jacobian matrix $\partial F / \partial y$. A Rosenbrock method is therefore fully described by the two matrices $\mathcal{A}=\left(\alpha_{i j}\right), \Gamma=\left(\gamma_{i j}\right)$ and the parameter $\gamma$.

Among the available methods are a second-order twostage method after Lanser et al. (2001).

$\mathbf{S} k_{1}=\tau F\left(y_{n}\right)$, 



Figure 4. Two neighbored cut cells with face and volume area information (left) and arrangement of face- and cell-centered tendency of momentum (right).

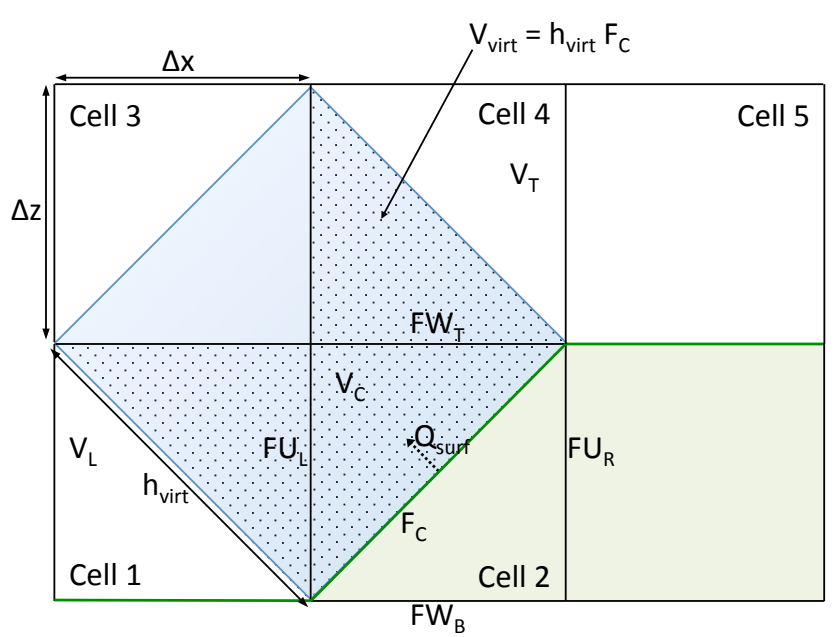

Figure 5. Example configuration for surface flux distribution around a cut cell. Green shading represents the solid part of the cells, whereas blue shading is the "virtual volume" normal to the cut cell face. The total surface flux $Q_{\text {surf }}$ is distributed within the dotted area.

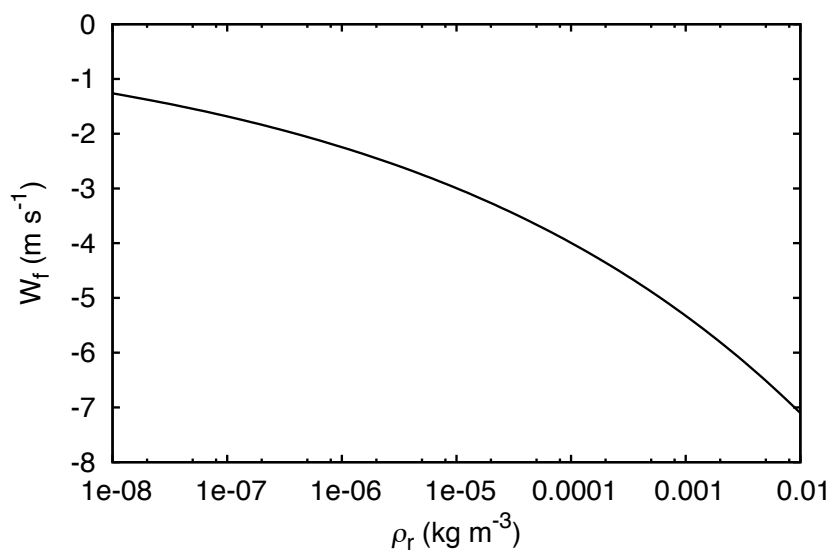

Figure 6. Terminal fall velocity of raindrops after Eq. (56).

$\mathbf{S} k_{2}=\tau F\left(y_{n}+\frac{2}{3} k_{1}\right)-\frac{4}{3} k_{1}$,

$y_{n+1}=y_{n}+\frac{5}{4} k_{1}+\frac{3}{4} k_{2}$,

$\mathbf{S}=\mathbf{I}-\gamma \tau \mathbf{J}, \quad \mathbf{J} \approx F^{\prime}\left(y_{n}\right)$,

with $\gamma=\frac{1}{2}+\frac{1}{6} \sqrt{3}$ or in matrix form in Table 2 .
Moreover, a new Rosenbrock method was constructed from a low storage three-stage second-order Runge-Kutta method, which is used in split-explicit time integration methods in the Weather Research and Forecasting (WRF) Model (Skamarock et al., 2008) or in the Consortium for Smallscale Modeling (COSMO) model (Doms et al., 2011). Its coefficients are given in Table 3.

The previously described Rosenbrock W methods allow a simplified solution of the linear systems without losing the order. When $\mathbf{J}=\mathbf{J}_{\mathrm{A}}+\mathbf{J}_{\mathrm{B}}$ the matrix $\mathbf{S}$ can be replaced by $\mathbf{S}=$ $\left(\mathbf{I}-\gamma \tau \mathbf{J}_{\mathrm{A}}\right)\left(\mathbf{I}-\gamma \tau \mathbf{J}_{\mathrm{B}}\right)$. Further simplification can be reached by omitting some parts of the Jacobian or by replacing of the derivatives by the same derivatives of a simplified operator $\widetilde{F}\left(w^{n}\right)$. For instance, higher-order interpolation formulae are replaced by the first-order upwind method. The structure of the Jacobian is

$\mathbf{J}=\left(\begin{array}{ccc}\frac{\partial F_{\rho}}{\partial \rho} & \frac{\partial F_{\rho}}{\partial \boldsymbol{V}} & 0 \\ \frac{\partial F_{\boldsymbol{V}}}{\partial \rho} & \frac{\partial F_{\boldsymbol{V}}}{\partial \boldsymbol{V}} & \frac{\partial F_{\boldsymbol{V}}}{\partial \Theta} \\ 0 & \frac{\partial F_{\Theta}}{\partial \boldsymbol{V}} & \frac{\partial F_{\Theta}}{\partial \Theta}\end{array}\right)$

A 0 block indicates that this block is not included in the Jacobian or is absent. The derivative with respect to $\rho$ is only taken for the buoyancy term in the vertical momentum equation. Note that this type of approximation is the standard approach in the derivation of the Boussinesq approximation starting from the compressible Euler equations. The matrix $\mathbf{J}$ can be decomposed as

$\mathbf{J}=\mathbf{J}_{\mathrm{T}}+\mathbf{J}_{\mathrm{P}}=\left(\begin{array}{ccc}\frac{\partial F_{\rho}}{\partial \rho} & 0 & 0 \\ \frac{\partial F_{\boldsymbol{V}}}{\partial \rho} & \frac{\partial F_{\boldsymbol{V}}}{\partial \boldsymbol{V}} & 0 \\ 0 & 0 & \frac{\partial F_{\Theta}}{\partial \Theta}\end{array}\right)+\left(\begin{array}{ccc}0 & \frac{\partial F_{\rho}}{\partial \boldsymbol{V}} & 0 \\ 0 & 0 & \frac{\partial F_{\boldsymbol{V}}}{\partial \Theta} \\ 0 & \frac{\partial F_{\Theta}}{\partial \boldsymbol{V}} & 0\end{array}\right)$

or

$\mathbf{J}=\mathbf{J}_{\mathrm{T}}+\mathbf{J}_{\mathrm{P}}=\left(\begin{array}{ccc}\frac{\partial F_{\rho}}{\partial \rho} & 0 & 0 \\ 0 & \frac{\partial F_{\boldsymbol{V}}}{\partial \boldsymbol{V}} & 0 \\ 0 & 0 & \frac{\partial F_{\Theta}}{\partial \Theta}\end{array}\right)+\left(\begin{array}{ccc}0 & \frac{\partial F_{\rho}}{\partial \boldsymbol{V}} & 0 \\ \frac{\partial F_{\boldsymbol{V}}}{\partial \rho} & 0 & \frac{\partial F_{\boldsymbol{V}}}{\partial \Theta} \\ 0 & \frac{\partial F_{\Theta}}{\partial \boldsymbol{V}} & 0\end{array}\right)$.

The first part of the splitting $\mathbf{J}_{\mathrm{T}}$ is called the transport/source part and contains the advection, diffusion and source terms like Coriolis, curvature, buoyancy, latent heat, and so on. 
The second matrix $\mathbf{J}_{\mathrm{P}}$ is called the pressure part and involves the derivatives of the pressure gradient, with respect to the density-weighted potential temperature, and of the divergence, with respect to momentum of the density and potential temperature equation. The difference between the two splitting approaches is the insertion of the derivative of the gravity term in the transport or pressure matrix. The first splitting (Eq. 25) damps sound waves. For this splitting the second linear system with the pressure part of the Jacobian can be reduced to a Poisson-like equation. The second splitting (Eq. 26) damps sound and gravity waves but the dimension of the pressure system is doubled. Both systems are solved by preconditioned conjugate-gradient (CG)-like methods (Dongarra et al., 1998).

For the transport/source system the Jacobian can be further split into

$\mathbf{J}_{\mathrm{T}}=\mathbf{J}_{\mathrm{AD}}+\mathbf{J}_{\mathrm{S}}$,

where the matrix $\mathbf{J}_{\mathrm{AD}}$ is the derivative of the advection and diffusion operator where the unknowns are coupled between grid cells. The matrix $\mathbf{J}_{\mathbf{S}}$ assembles the source terms. Here the coupling is between the unknowns of different components in each grid cell. With this additional splitting the linear equation

$\left(\mathbf{I}-\gamma \tau \mathbf{J}_{\mathrm{AD}}-\gamma \tau \mathbf{J}_{\mathrm{S}}\right) \Delta w=R$

is preconditioned from the right with the matrix

$\mathbf{P}_{\mathrm{r}}=\left(\mathbf{I}-\gamma \tau \mathbf{J}_{\mathrm{AD}}\right)^{-1}$

and from the left with the matrix

$\mathbf{P}_{1}=\left(\mathbf{I}-\gamma \tau \mathbf{J}_{\mathbf{S}}\right)^{-1}$

The matrix

$\mathbf{P}_{1}\left(\mathbf{I}-\gamma \tau \mathbf{J}_{\mathrm{AD}}-\gamma \tau \mathbf{J}_{\mathrm{S}}\right) \mathbf{P}_{\mathrm{r}}$

can be written in the following form by using the Eisenstat trick (Eisenstat, 1981):

$\left(\mathbf{I}-\gamma \tau \mathbf{P}_{1} \mathbf{J}_{\mathrm{AD}}\right) \mathbf{P}_{\mathrm{r}}=\left(\mathbf{I}+\mathbf{P}_{1}\left(\left(\mathbf{I}-\gamma \tau \mathbf{J}_{\mathrm{AD}}\right)+\mathbf{I}\right)\right) \mathbf{P}_{\mathrm{r}}$.

Therefore, only the LU-decomposition of the matrix (I $\left.\gamma \tau \mathbf{J}_{\mathrm{S}}\right)$ has to be stored. The matrix $\left(\mathbf{I}-\gamma \tau \mathbf{J}_{\mathrm{AD}}\right)$ is inverted by a fixed number of Gauss-Seidel iterations.

The second matrix of the splitting approach is written in case of the first splitting (Eq. 25) as follows:

$\left(\mathbf{I}-\gamma \tau \mathbf{J}_{\mathrm{P}}\right)=\left(\begin{array}{cc}\mathbf{I} & \gamma \tau \operatorname{GRADD}_{\Theta} \\ \gamma \tau \mathrm{DIV}_{\boldsymbol{V}} & \mathbf{I}\end{array}\right)$,

where $\mathbf{D}_{V}$ and $\mathbf{D}_{\Theta}$ are diagonal matrices. GRAD and DIV are matrix representations of the discrete gradient and divergence operator. The entries of the matrix $\mathbf{D}_{V}$ are the potential temperature at cell faces and the entries of the matrix $\mathbf{D}_{\Theta}$ are the derivative of the pressure in the cell center with respect to the density-weighted potential temperature. Elimination of the momentum part gives a Helmholtz equation for the increment of the potential temperature. This equation is solved by a CG-method with a multi-grid as a preconditioner. For the second splitting (Eq. 26), the resulting matrix is doubled in dimension and not symmetric anymore.

As basic iterative solvers, BiCGStab is applied for the transport/source system and GMRES for the pressure part (Dongarra et al., 1998). The number of iterations for the two iterative methods are problem dependent. They increase with increasing time step and are usually in the range of $2-5$ iterations.

\section{Physical parameterizations}

\subsection{Smagorinsky subgrid-scale model}

The set of coupled differential equations can be solved for a given flow problem. For simulating turbulent flows with large eddy simulations, the Euler equations mentioned at the beginning have to be modified. Within the technique of LES it is necessary to characterize the unresolved motion. By solving Eqs. (1)-(3) numerically with a grid size which is larger than the size of the smallest turbulent scales, the equations have to be filtered. Large eddy simulation employs a spatial filter to separate the large-scale motion from the small scales. Large eddies are resolved explicitly by the prognostic Euler equations down to a predefined filter-scale $\Delta$, while smaller scales have to be modeled. Due to the filtering operation, additional terms that cannot be derived trivially occur in the set of Euler equations.

Nevertheless, to solve the filtered set of equations, it is necessary to parameterize the additional subgrid-scale stress terms $\tau_{i j}=\overline{u_{i} u_{j}}-\bar{u}_{i} \bar{u}_{j}$ for momentum and $q_{i j}=\overline{u_{i} q_{j}}-\bar{u}_{i} \bar{q}_{j}$ for potential. Note that $\tau_{i j}$ expresses the effect of subgridscale motion on the resolved large scales and is often represented as an additional viscosity $v_{\mathrm{t}}$ with the following formulation:

$\tau_{i j}=-2 v_{\mathrm{t}} \bar{S}_{i j}$,

where $\bar{S}_{i j}=\frac{1}{2}\left(\frac{\partial \bar{u}_{i}}{\partial x_{j}}+\frac{\partial \bar{u}_{j}}{\partial x_{i}}\right)$ is the strain rate tensor and $v_{\mathrm{t}}$ the turbulent eddy viscosity. To determine the additional eddy viscosity, the standard Smagorinsky subgrid-scale model (Smagorinsky, 1963) is used:

$v_{\mathrm{t}}=\left(C_{\mathrm{s}} \bar{\Delta}\right)^{2}|\overline{\mathbf{S}}|$,

where $\bar{\Delta}$ is a length scale, $C_{\mathrm{s}}$ the Smagorinsky coefficient, and using the Einstein summation notation for standardization $|\overline{\mathbf{S}}|=\sqrt{2 \bar{S}_{i j} \bar{S}_{i j}}$. The grid spacing is used as a measure for the length scale. This standard Smagorinsky subgridscale model is widely used in atmospheric and engineering 
applications. The Smagorinsky coefficient $C_{\mathrm{s}}$ has a theoretical value of about 0.2 , as estimated by Lilly (1967). Applying this value to a turbulence-driven flow with thermal convection fields results in a good agreement with observations as shown by Deardorff (1972).

To take stratification effects into account, Lilly (1962) modified the standard Smagorinsky formulation by changing the eddy viscosity to

$v_{\mathrm{t}}=\left(C_{\mathrm{s}} \bar{\Delta}\right)^{2} \max \left[0,\left(|\overline{\mathbf{S}}|^{2}\left(1-\frac{R i}{P r}\right)\right)\right]^{1 / 2}$

with

$R i=\frac{\frac{g}{\theta_{\rho}} \frac{\partial \theta_{\rho}}{\partial z}}{|\overline{\mathbf{S}}|^{2}}$.

Here, $R i$ is the Richardson number and $P r$ is the turbulent Prandtl number. In a stable boundary layer the vertical gradient of the potential temperature is greater than zero (positive), which leads to a positive Richardson number and, thus, the additional term $R i / P r$ reduces the square of the strain rate tensor and decreases the turbulent eddy viscosity. Therefore, less turbulent vertical mixing takes place.

The implementation in the ASAM code is accomplished in the main diffusion routine of the model. It develops the whole term of $\partial / \partial x_{j}\left[\rho D S_{i j}\right]$ for every time step. The coefficient $D$ represents $D_{\text {mom }}$ for the momentum and $D_{\text {pot }}$ for the potential subgrid-scale stress. Further routines describe the computation of $D_{\text {mom }}$ and $D_{\text {pot }}$ in the following way:

$D_{\text {mom }}=\left(C_{\mathrm{s}} \bar{\Delta}\right)^{2}|\overline{\mathbf{S}}|$.

The potential subgrid-scale stress is related to the Prandtl similarity and can be developed by dividing the subgrid-scale stress tensor for momentum by the turbulent Prandtl number $\operatorname{Pr}$ that typically has a value of $1 / 3$ (Deardorff, 1972). The length scale $\bar{\Delta}$ in the standard Smagorinsky formulation is set to the value of grid spacing. However, the cut cell approach makes it difficult because of tiny and/or anisotropic cells. To overcome this deficit the value is defined after Scotti et al. (1993):

$\bar{\Delta}=\left(\Delta_{1} \Delta_{2} \Delta_{3}\right)^{1 / 3} f\left(a_{1}, a_{2}\right)$.

$\Delta$ is the grid spacing in orthogonal directions, and a correction function $f$ is applied as follows:

$$
\begin{aligned}
& f\left(a_{1}, a_{2}\right)=\cosh \left[\frac{4}{27}\left(\ln ^{2} a_{1}-\ln a_{1} \ln a_{2}+\ln ^{2} a_{2}\right)\right]^{1 / 2} \\
& \text { with } \quad a_{1}=\frac{\Delta_{1}}{\Delta_{3}}, \quad a_{2}=\frac{\Delta_{2}}{\Delta_{3}} .
\end{aligned}
$$

Here, $a_{1}$ and $a_{2}$ are the ratios of grid spacing in different directions with the assumption that $\Delta_{1} \leq \Delta_{2} \leq \Delta_{3}$. For an isotropic grid $f=1$.

\subsection{Two-moment warm cloud microphysics scheme}

The implemented microphysics scheme is based on the work of Seifert and Beheng (2006). This scheme explicitly represents two moments (mass and number density) of the hydrometeor classes cloud droplets and rain drops. Ice phase hydrometeors are currently not implemented in the model. Altogether, seven microphysical processes are included: condensation/evaporation (COND), cloud condensation nuclei $(\mathrm{CCN})$ activation to cloud droplets at supersaturated conditions (ACT), autoconversion (AUTO), self-collection of cloud droplets (SCC), self-collection of rain drops (SCR), accretion (ACC) and evaporation of rain (EVAP):

$$
\begin{aligned}
& \frac{\partial\left(\rho q_{\mathrm{v}}\right)}{\partial t}+\nabla \cdot\left(\rho \boldsymbol{v} q_{\mathrm{v}}\right)=-S_{\mathrm{COND}}-S_{\mathrm{ACT}}+S_{\mathrm{EVAP}}, \\
& \frac{\partial\left(\rho q_{\mathrm{c}}\right)}{\partial t}+\nabla \cdot\left(\rho \boldsymbol{v} q_{\mathrm{c}}\right)=+S_{\mathrm{COND}}+S_{\mathrm{ACT}}-S_{\mathrm{AUTO}}-S_{\mathrm{ACC}} \\
& \frac{\partial\left(\rho q_{\mathrm{r}}\right)}{\partial t}+\nabla \cdot\left(\rho \boldsymbol{v} q_{\mathrm{r}}\right)=+S_{\mathrm{AUTO}}+S_{\mathrm{ACC}}-S_{\mathrm{EVAP}}, \\
& \frac{\partial N_{\mathrm{CCN}}}{\partial t}+\nabla \cdot\left(\boldsymbol{v} N_{\mathrm{CCN}}\right)=-S_{\mathrm{COND}_{\mathrm{N}}}-S_{\mathrm{ACT}_{\mathrm{N}}}+S_{\mathrm{EVAP}_{\mathrm{N}}}, \\
& \frac{\partial N_{\mathrm{c}}}{\partial t}+\nabla \cdot\left(\boldsymbol{v} N_{\mathrm{c}}\right)=+S_{\mathrm{COND}_{\mathrm{N}}}+S_{\mathrm{ACT}_{\mathrm{N}}}-S_{\mathrm{AUTO}_{\mathrm{N}}} \\
& \quad-S_{\mathrm{ACC}_{\mathrm{N}}}-S_{\mathrm{SCC}}, \\
& \frac{\partial N_{\mathrm{r}}}{\partial t}+\nabla \cdot\left(\boldsymbol{v} N_{\mathrm{r}}\right)=+S_{\mathrm{AUTO}_{\mathrm{N}}}+S_{\mathrm{ACC}_{\mathrm{N}}}-S_{\mathrm{EVAP}_{\mathrm{N}}}-S_{\mathrm{SCR}} .
\end{aligned}
$$

Details on the conversion rates can be found in Seifert and Beheng (2006). Additionally, a limiter function is used to ensure numerical stability and avoid nonphysical negative values (Horn, 2012). Since there is no saturation adjustment technique in ASAM, the condensation process is taken as an example to demonstrate the physical meaning of the limiter functions. Considering the available water vapor density $\rho_{\mathrm{v}}$ and the cloud water density $\rho_{\mathrm{c}}$, the process of condensation (or evaporation of cloud water, respectively) is forced by the water vapor density deficit and limited by the available cloud water.

$$
\begin{aligned}
& \mathrm{FOR}=\rho_{\mathrm{v}}-\left(p_{\mathrm{vs}} T / R_{\mathrm{v}}\right) \\
& \mathrm{LIM}=\rho_{\mathrm{c}} \\
& S_{\mathrm{COND}}=\frac{\mathrm{FOR}-\mathrm{LIM}+\left(\mathrm{FOR}^{2}+\mathrm{LIM}^{2}\right)^{1 / 2}}{\tau_{\mathrm{COND}}}
\end{aligned}
$$

Here, $p_{\mathrm{vs}}$ is the saturation vapor pressure and the relaxation time is set to $\tau_{\mathrm{COND}}=1 \mathrm{~s}$. The numerator term is called the Fischer-Burmeister function and has originally been used in the optimization of complementary problems (cf. Kong et al., 2010). A simple model after Horn (2012) is applied to determine the corresponding changes in the number concentrations and to ensure a reduction of the cloud droplet number density to zero if there is no cloud water present. This means that $N_{\mathrm{c}}$ decreases when droplets are getting too small,

$$
S_{\mathrm{COND}_{\mathrm{N}}}=\min \left(0, C\left[\frac{\rho_{\mathrm{c}}}{x_{\mathrm{min}}}-N_{\mathrm{c}}\right]\right) \text {, }
$$


and increases when droplets are getting too large,

$S_{\mathrm{COND}_{\mathrm{N}}}=\max \left(0, C\left[\frac{\rho_{\mathrm{c}}}{x_{\mathrm{max}}}-N_{\mathrm{c}}\right]\right)$,

where $x_{\min }$ and $x_{\max }$ are limiting parameters for cloud water. This ensures that the cloud droplet number concentration is within a certain range defined by distribution parameters in Seifert and Beheng (2006) if condensate is present. A timescale factor of $C=0.01 \mathrm{~s}^{-1}$ controls the speed of this correction and appears to be reasonable for this particular process.

\subsection{Precipitation}

The sedimentation velocity of raindrops is derived as in the operationally used COSMO model from the German Weather Service (Doms et al., 2011). There, the following assumptions are made. The precipitation particles are exponentially distributed with respect to their drop diameter (Marshall-Palmer distribution):

$f_{\mathrm{r}}(D)=N_{0}^{\mathrm{r}} \exp -\lambda_{\mathrm{r}} D$

Here, $\lambda_{\mathrm{r}}$ is the slope parameter of the distribution function and $N_{0}^{\mathrm{r}}=8 \times 10^{6} \mathrm{~m}^{-4}$ is an empirically determined distribution parameter. The terminal fall velocity of raindrops is then assumed to be uniquely related to drop size, which is expressed by the following empirical function:

$W_{\mathrm{f}}(D)=c_{\mathrm{r}} D^{1 / 2}$

with $c_{\mathrm{r}}=130 \mathrm{~m}^{1 / 2} \mathrm{~s}^{-1}$. Finally, the precipitation flux of rainwater can be calculated as

$\mathbf{P}_{\mathrm{r}}=\rho_{\mathrm{r}} W_{\mathrm{f}}\left(\rho_{\mathrm{r}}\right)=\int_{0}^{\infty} m(D) W_{\mathrm{f}}(D) f_{\mathrm{r}}(D) \mathrm{d} D$

with the raindrop mass

$m(D)=\pi \rho_{\mathrm{W}} D^{3} / 6$,

where $\rho_{\mathrm{W}}=1000 \mathrm{~kg} \mathrm{~m}^{-3}$ is the mass density of water. This leads to an expression for the terminal fall velocity of raindrops in dependence on their density:

$W_{\mathrm{f}}\left(\rho_{\mathrm{r}}\right)=-c_{\mathrm{r}} \frac{\Gamma(4,5)}{6}\left(\frac{\rho_{\mathrm{r}}}{\pi \rho_{\mathrm{W}} N_{0 r}}\right)^{1 / 8}$.

This takes place at the tendency equation for the rain water density:

$\frac{\partial\left(\rho q_{\mathrm{r}}\right)}{\partial t}+\nabla_{h} \cdot\left(\rho \boldsymbol{v}_{h} q_{\mathrm{r}}\right)+\frac{\partial}{\partial z}\left(\rho q_{\mathrm{r}}\left[w+W_{\mathrm{f}}\right]\right)=S_{q_{\mathrm{r}}}$

\subsection{Surface fluxes}

A simple way to parameterize surface heat fluxes is the usage of a constant flux layer. There, the energy flux is directly given and does not depend on other variables. With the density potential temperature formulation (Eq. 3), the source term for this quantity has to be calculated:

$$
\begin{aligned}
\frac{\partial\left(\rho \theta_{\rho}\right)}{\partial t}+\frac{\partial}{\partial x_{j}}\left(\rho \theta_{\rho} u_{j}\right) & =\rho \frac{\partial \theta_{\rho}}{\partial t}+\theta_{\rho} \frac{\partial \rho}{\partial t}+\theta_{\rho} \frac{\partial \rho u_{j}}{\partial x_{j}}+\rho u_{j} \frac{\partial \theta_{\rho}}{\partial x_{j}} \\
& =\rho\left(\frac{\partial \theta_{\rho}}{\partial t}+u_{j} \frac{\partial \theta_{\rho}}{\partial x_{j}}\right)+\theta_{\rho}\left(\frac{\partial \rho}{\partial t}+\frac{\partial \rho u_{j}}{\partial x_{j}}\right) \\
& =\rho \frac{\mathrm{d} \theta_{\rho}}{\mathrm{d} t}+\theta_{\rho} S_{\mathrm{v}} .
\end{aligned}
$$

$S_{\mathrm{V}}$ is the source term of water vapor (in $\mathrm{kg} \mathrm{m}^{-3} \mathrm{~s}^{-1}$ ). Considering Eq. (A33), adding the sensible heat flux and neglecting phase changes leads to

$\frac{\partial\left(\rho \theta_{\rho}\right)}{\partial t}+\frac{\partial}{\partial x_{j}}\left(\rho \theta_{\rho} u_{j}\right)=S_{\theta_{\rho}}$

with

$S_{\theta_{\rho}}=\rho \theta_{\rho}\left(\frac{S_{\mathrm{h}}}{T}+\frac{S_{\mathrm{v}}}{\rho_{\mathrm{d}}}\left[\frac{R_{\mathrm{v}}}{R_{\mathrm{m}}}-\ln \pi\left(\frac{R_{\mathrm{v}}}{R_{\mathrm{m}}}-\frac{c_{\mathrm{pv}}}{c_{\mathrm{pml}}}\right)\right]\right)$,

where $S_{\mathrm{h}}$ is the heat source (in $\mathrm{K} \mathrm{s}^{-1}$ ), $R_{\mathrm{m}}=R_{\mathrm{d}}+r_{\mathrm{v}} R_{\mathrm{V}}$ and $c_{\mathrm{pml}}=c_{\mathrm{pd}}+r_{\mathrm{v}} c_{\mathrm{pv}}+r_{1} c_{\mathrm{pl}}$ are the gas constant and the specific heat capacity for the air mixture, respectively. The corresponding surface fluxes (in $\mathrm{W} \mathrm{m}^{-2}$ ) are

$S_{\mathrm{sens}}=S_{\mathrm{h}} \frac{\rho_{\mathrm{d}} c_{\mathrm{pml}}}{\rho A}$,

$S_{\text {lat }}=S_{\mathrm{v}} L_{\mathrm{v}}(T) \frac{V}{A}$

Here, $L_{\mathrm{v}}=L_{00}+\left(c_{\mathrm{pv}}-c_{\mathrm{pl}}\right) T$ is the latent heat of vaporization, $A$ is the cell surface at the bottom boundary and $V$ the cell volume.

\subsection{Soil model}

In order to account for the interaction between land and atmosphere and the high diurnal variability of the meteorological variables in the surface layer, a soil model has been implemented into ASAM. In contrast to the constant flux layer model, the computation of the heat and moisture fluxes are now dependent on radiation, evaporation and the transpiration of vegetated area. Phase changes are not covered yet and intercepted water is only considered in liquid state.

The implemented surface flux scheme follows the description of Jiménez et al. (2012), which is the revised flux scheme used in the WRF model. The surface fluxes of momentum, heat and moisture are parameterized in the following way, respectively:

$\tau_{z x}=\rho C_{\mathrm{m}}\left|\boldsymbol{v}_{\boldsymbol{h}}\right| u(h)$, 
$-\rho c_{p} \overline{w^{\prime} \theta^{\prime}}=\rho c_{p} C_{\mathrm{h}}\left|\boldsymbol{v}_{\boldsymbol{h}}\right|\left(\theta(h)-\theta\left(z_{0 T}\right)\right)$,

$-\rho L \overline{w^{\prime} q^{\prime}}=\rho L C_{\mathrm{q}}\left|\boldsymbol{v}_{\boldsymbol{h}}\right|\left(q(h)-q\left(z_{0 q}\right)\right)$.

$C_{\mathrm{m}}, C_{\mathrm{h}}$ and $C_{\mathrm{q}}$ are the bulk transfer coefficients and it is considered that $C_{\mathrm{h}}=C_{\mathrm{q}}$. In Jiménez et al. (2012), the bulk transfer coefficients are defined as follows:

$C_{\mathrm{m}, \mathrm{h}}=\frac{k^{2}}{\Psi_{\mathrm{M}} \Psi_{\mathrm{M}, \mathrm{H}}}$

with

$\Psi_{\mathrm{M}, \mathrm{H}}=\ln \left(\frac{z+z_{0}}{z_{0}}\right)-\phi_{\mathrm{m}, \mathrm{h}}\left(\frac{z+z_{0}}{L}\right)+\phi_{\mathrm{m}, \mathrm{h}}\left(\frac{z_{0}}{L}\right)$

and $\phi_{\mathrm{m}, \mathrm{h}}$ representing the integrated similarity functions. $L$ stands for the Obukhov length and $k$ is the von Kármán constant. In neutral to highly stable conditions $\phi_{\mathrm{m}, \mathrm{h}}$ follows Cheng and Brutsaert (2005) and in unstable situations the $\phi$ functions follow Fairall et al. (1996). For further details concerning limitations and restrictions see Jiménez et al. (2012).

The transport of the soil water as a result of hydraulic pressure due to diffusion and gravity within the soil layers is described by Richard's equation:

$\frac{\partial W_{\text {soil }, k}}{\partial t}=\frac{\partial}{\partial z}\left(D_{\text {iff }} \frac{\partial W_{\text {soil }, k}}{\partial z}+\kappa_{\text {soil }, k}\right)$

with the diffusion coefficient

$D_{\text {iff }}=\kappa_{\text {soil }, k} \frac{\partial \Psi_{\text {soil }, k}}{\partial W_{\text {soil }, k}}$.

$W_{\text {soil, } k}$ is the volumetric water content in the $k$ th soil layer.

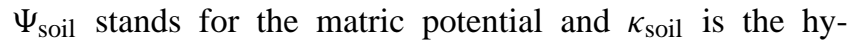
draulic conductivity. $\Psi_{\text {soil }}$ and $\kappa_{\text {soil }}$ are parameterized based on Van Genuchten (1980):

$\kappa_{\text {soil }}=\kappa_{\text {sat }} \sqrt{W_{\text {eff }}}\left(1-\left[1-\left(W_{\text {eff }}\right)^{\frac{1}{m}}\right]^{m}\right)^{2^{\prime}}$

$\Psi_{\text {soil }}=\Psi_{\text {sat }}\left[\left(W_{\text {eff }}\right)^{-\frac{1}{m}}-1\right]^{\frac{1}{n}}$.

$W_{\text {eff }}$ describes the effective soil wetness, which takes a residual water content $W_{\text {res }}$ into account, restricting the soil from complete desiccation. $\kappa_{\text {sat }}$ and $\Psi_{\text {sat }}$ are the hydraulic conductivity and the matric potential at saturated conditions, respectively. The parameters $m$ and $n$ describe the pore distribution (Braun, 2002) with $m=1-1 / n$ (also see Tables B1 and B2).

Further addition/extraction of soil water is controlled by the percolation of intercepted water into the ground and the evaporation and transpiration of water from bare soil and vegetation. The mechanisms implemented are based on the Multi-Layer Soil and Vegetation Model TERRA_ML as described in Doms et al. (2011). The evaporation of bare soil is adjusted to the parameterization proposed by Noilhan and
Planton (1989). The variation of the soil temperature is a result of heat conductivity depending on the soil texture and the soil water content of the respective soil layer:

$\frac{\partial T_{\text {soil }}}{\partial t}=\frac{1}{\rho c} \frac{\partial}{\partial z}\left[\lambda \frac{\partial T_{\text {soil }}}{\partial z}+E_{q} \rho_{\mathrm{w}} c_{\mathrm{W}} \bar{T}_{\text {soil }}\right]$.

$T_{\text {soil }}$ is the absolute temperature in the $k$ th soil layer (in $\mathrm{K}$ ), $\bar{T}_{\text {soil }}$ is the mean soil temperature of two neighboring soil layers. The change in internal energy due to changes in moisture by the inner soil water flux, evapotranspiration and evaporation from the upper soil layer and the interception reservoir is treated by the second term in brackets. The heat conductivity $\lambda$ and the volumetric heat capacity $\rho c$ are variables that depend on the soil texture. The heat capacity of the soil $\rho c$ formulated by Chen and Dudhia (2001) is the sum of the heat capacity of dry soil $\left(\rho_{0} c_{0}\right.$, see Tables B1 and B2), the heat capacity of wet soil $\left(\rho_{\mathrm{w}} c_{\mathrm{w}}\right)$ and the heat capacity of the air within the soil pores $\left(\rho_{\mathrm{a}} c_{\mathrm{a}}\right)$.

$\rho c=W_{\text {soil }} \rho_{\mathrm{w}} c_{\mathrm{W}}+\left(1-W_{\mathrm{pv}}\right) \rho_{0} c_{0}+\left(W_{\mathrm{pv}}-W_{\text {soil }}\right) \rho_{\mathrm{a}} c_{\mathrm{a}}$

with $W_{\mathrm{pv}}$ corresponding to the soil pores, $\rho_{\mathrm{W}} c_{\mathrm{W}}=4.18 \times$ $10^{6} \mathrm{~J} \mathrm{~m}^{-3} \mathrm{~K}^{-1}$ and $\rho_{\mathrm{a}} c_{\mathrm{a}}=1298 \mathrm{~J} \mathrm{~m}^{-3} \mathrm{~K}^{-1}$. The heat conductivity $\lambda$ is defined after Pielke (1984):

$\lambda= \begin{cases}418 \exp \left\{-\Psi_{\log }-2.7\right\} & \text { if } \Psi_{\log } \leq 5.1 \\ 0.172 & \text { if } \Psi_{\log }>5.1\end{cases}$

with $\Psi_{\log }=\log _{10}\left|100 \Psi_{\text {soil }}\right|$.

The topmost layer is exposed to the incoming radiation and thus has the strongest variation in temperature in comparison to the other soil layers within the ground. The temperature equation of the first layer is, in addition to the incoming radiation, determined by the latent and sensible heat flux.

$\frac{\partial T_{\text {soil }, 1}}{\partial t}=\frac{1}{\rho c} \frac{\partial}{\partial z}\left[\left(\lambda \frac{\partial T_{\text {soil }, 1}}{\partial z}\right)+\Delta Q\right]$

with

$\Delta Q=Q_{\mathrm{dir}}+Q_{\mathrm{dif}}-\sigma T_{\mathrm{sfc}}^{4}-c_{p} Q_{\mathrm{SH}}-L_{\mathrm{v}} Q_{\mathrm{LH}} \cdot$

Here, $Q_{\mathrm{LH}}$ is the latent heat flux, describing the moisture flux between soil and atmosphere as the sum of evaporation and transpiration and $Q_{\mathrm{SH}}$ is the sensible heat flux. $Q_{\mathrm{dir}}$ and $Q_{\mathrm{dif}}$ represent the direct and diffusive irradiation, respectively.

\section{Test cases}

In this section, we present six example test cases. In five of these cases, orography or obstacles are included to test conservation properties and model accuracy. The first test case is the rising heat bubble prescribed in Wicker and Skamarock 

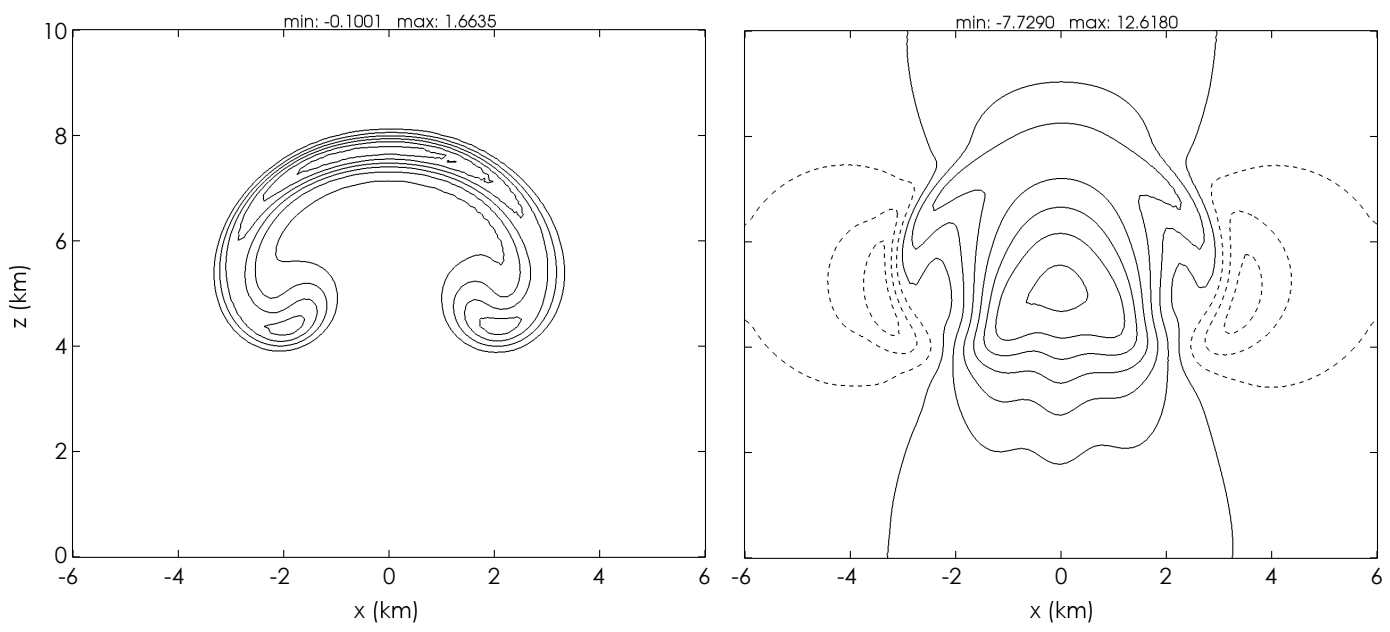

Figure 7. Results for the dry bubble simulation after Wicker and Skamarock (1998) at $t=1000 \mathrm{~s}$ : contours of perturbation potential temperature (left panel) and vertical velocity (right panel). Solid (dashed) lines indicate positive (negative) values. Contour intervals are $0.25 \mathrm{~K}$ (zero contour omitted) and $2 \mathrm{~ms}^{-1}$, respectively.

(1998). The bubble is initially defined by a radial temperature perturbation, which leads to rising motion due to buoyant forces. Results of a two-dimensional gravity wave simulation (Schaer et al., 2002) are shown in the second test case. There, the gravity waves are induced by an idealized mountain ridge. Two subcases with different atmospheric stabilities and reference temperatures are examined. The third case is a sinking cold bubble in a dry environment, from which a density current develops (Straka et al., 1993). A $1 \mathrm{~km}$ tall hill is added at the left side of the domain so that the resulting current overflows over the mountain. Considering moisture effects and phase changes, the moist bubble case by Bryan and Fritsch (2002) with the addition of a mid-air zeppelin (Klein et al., 2009; Jebens et al., 2011) is performed. Besides analyzing the flow field in the vicinity of the obstacles, conservation studies regarding total energy are performed for both cases. Another idealized benchmark case is carried out to analyze the accuracy of the presented discretization method for cut cells. There, a scalar field is advected by a radial wind field in an annulus (Berger and Helzel, 2012). This is also a suitable test for convergence studies by calculating $L_{1}$ and $L_{\infty}$ error norms since an analytical solution can be used for comparison. The last test case is a three-dimensional simulation study regarding flow dynamics around an idealized mountain and orographic precipitation by Kunz and Wassermann (2011).

\subsection{Dry bubble}

A two-dimensional simulation of a rising thermal is presented in Wicker and Skamarock (1998). This test case is also used as a dry reference case for the moist bubble simulation in Bryan and Fritsch (2002). The domain is $20 \mathrm{~km}$ in the horizontal direction and $10 \mathrm{~km}$ in the vertical direction with a uniform grid spacing of $125 \mathrm{~m}$. A mean flow of $U=20 \mathrm{~m} \mathrm{~s}^{-1}$ is applied. After $1000 \mathrm{~s}$ the bubble has been transported through the lateral periodic boundaries and is again located at the center of the domain. The perturbation field takes the following form:

$\theta^{\prime}=2 \cos ^{2}\left(\frac{\pi L}{2}\right)$

with

$L=\sqrt{\left(\frac{x-x_{\mathrm{c}}}{x_{\mathrm{r}}}\right)^{2}+\left(\frac{z-z_{\mathrm{c}}}{z_{\mathrm{r}}}\right)^{2}} \leq 1$.

The parameters $x_{\mathrm{c}}=10 \mathrm{~km}, z_{\mathrm{c}}=2 \mathrm{~km}$ and $x_{\mathrm{r}}=z_{\mathrm{r}}=2 \mathrm{~km}$ determine the position and radius of the heat bubble. The atmosphere is in hydrostatic balance and neutrally stable with a surface pressure $p_{0}=1000 \mathrm{hPa}$ and a constant potential temperature $\theta=300 \mathrm{~K}$. Results of this simulation are displayed in Fig. 7. The overall shape is reproduced and comparable to the reference solution. Slight asymmetries are observed due to lateral transport. However, it becomes apparent that third-order Runge-Kutta time integration together with a fifth-order advection scheme produces better results in terms of minimum/maximum values and symmetry (cf. Bryan and Fritsch, 2002) than the third-order advection scheme that is used here.

\subsection{2-D mountain gravity waves}

In this test case, a flow over a mountain ridge is simulated (Schaer et al., 2002). Skamarock et al. (2012) A dry stable atmosphere is defined by a constant Brunt-Väisälä frequency $N$, upstream surface temperature $T_{0}$, surface pressure $p_{0}$ and a uniform inflow velocity $U$. The domain extends $200 \mathrm{~km}$ horizontally and $19.5 \mathrm{~km}$ vertically with grid spacings of $\Delta x=500 \mathrm{~m}$ and $\Delta z=300 \mathrm{~m}$. The structure of 

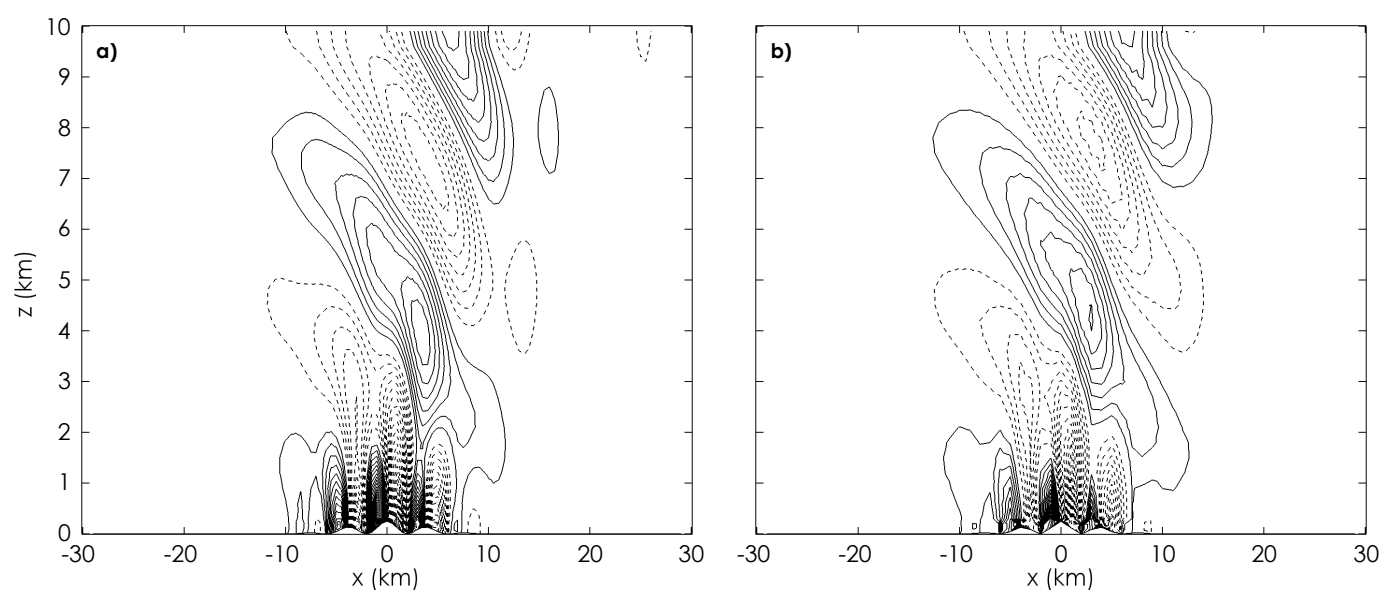

Figure 8. Steady-state solution for the simulation of the Schaer et al. (2002) test case: contours of vertical velocity for (a) $N=0.01 \mathrm{~s}^{-1}$ and (b) $N=0.01871 \mathrm{~s}^{-1}$. Solid (dashed) lines indicate positive (negative) values. Contour intervals are (a) $0.05 \mathrm{~m} \mathrm{~s}^{-1}$ and (b) $0.09355 \mathrm{~m} \mathrm{~s}^{-1}$, respectively.

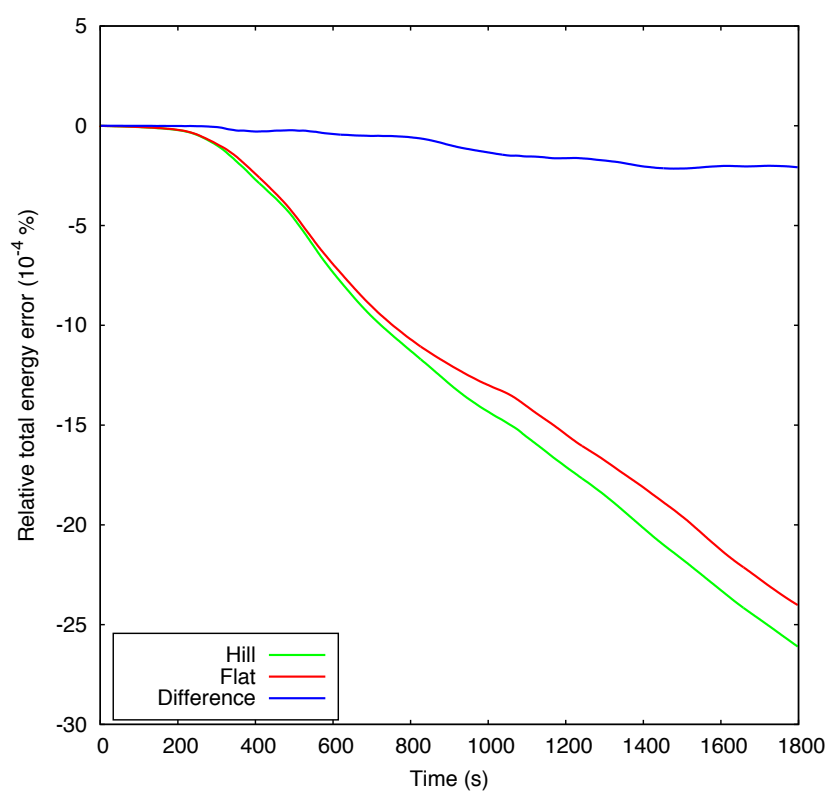

Figure 9. Time series of total energy error for the density current test case with and without the hill. The error is expressed as $10^{-4} \%$ of the total energy at the beginning of the simulation.

the mountain ridge is represented by a bell curve shape with superposed variations:

$h(x)=h_{0} \exp \left(-[x / a]^{2}\right) \cos ^{2}(\pi x / \lambda)$,

where $h_{0}=250 \mathrm{~m}, a=5 \mathrm{~km}$ and $\lambda=4 \mathrm{~km}$. The simulation result for the steady state is shown in Fig. 8. There are no nonphysical distorted wave patterns and the result agrees well with the analytical and reference solutions shown in Schaer et al. (2002).

\subsection{Cold bubble with orography interaction}

A nonlinear test problem is the density current simulation study documented in Straka et al. (1993). In this case, the computational domain extends from -18 to $18 \mathrm{~km}$ in the horizontal direction and from 0 to $6.4 \mathrm{~km}$ in the vertical direction with isotropic grid spacing of $\Delta x=\Delta z=100 \mathrm{~m}$. Boundary conditions are periodic in the $x$ direction and the free-slip condition is applied for the top and bottom model boundaries. The total integration time is $t=1800 \mathrm{~s}$. The initial atmosphere is in a dry and hydrostatically balanced state and there is a horizontally homogeneous environment with $\bar{\theta}=300 \mathrm{~K}$ (i.e., neutrally stratified). The perturbation (cold bubble with negative buoyancy) is defined by a temperature perturbation of

$T^{\prime}=\left\{\begin{array}{ll}0.0^{\circ} \mathrm{C} & \text { if } L>1.0 \\ -15.0^{\circ} \mathrm{C}(\cos [\pi L]+1.0) / 2 & \text { if } L \leq 1.0\end{array}\right.$,

where

$L=\left(\left[\left(x-x_{\mathrm{c}}\right) x_{\mathrm{r}}^{-1}\right]^{2}+\left[\left(z-z_{\mathrm{c}}\right) z_{\mathrm{r}}^{-1}\right]^{2}\right)^{0.5}$

and $x_{\mathrm{c}}=0.0 \mathrm{~km}, x_{\mathrm{r}}=4.0 \mathrm{~km}, z_{\mathrm{c}}=3.0 \mathrm{~km}$ and $z_{\mathrm{r}}=2.0 \mathrm{~km}$. At first, there is no fixed physical viscosity turned on as in the original test case (with $v=75 \mathrm{~m}^{2} \mathrm{~s}^{-1}$ ) since a conservation test regarding total energy is carried out. For this test, two simulation runs are performed with (a) the above described standard setup and (b) a modified setup where a mountain is added at the left part of the domain. The mountain follows the "Witch of Agnesi" curve:

$h(x)=\left\{\begin{array}{ll}H /\left(1+\left[\left(x-x_{\mathrm{M}}\right) / a_{1}\right]^{2}\right) & \text { if } x<x_{\mathrm{M}} \\ H /\left(1+\left[\left(x-x_{\mathrm{M}}\right) / a_{2}\right]^{2}\right) & \text { if } x \geq x_{\mathrm{M}}\end{array}\right.$,

with half-width lengths $a_{1}=a_{2}=1 \mathrm{~km}$, mountain peak center position $x_{\mathrm{M}}=-6 \mathrm{~km}$ and mountain height $H=1 \mathrm{~km}$. 

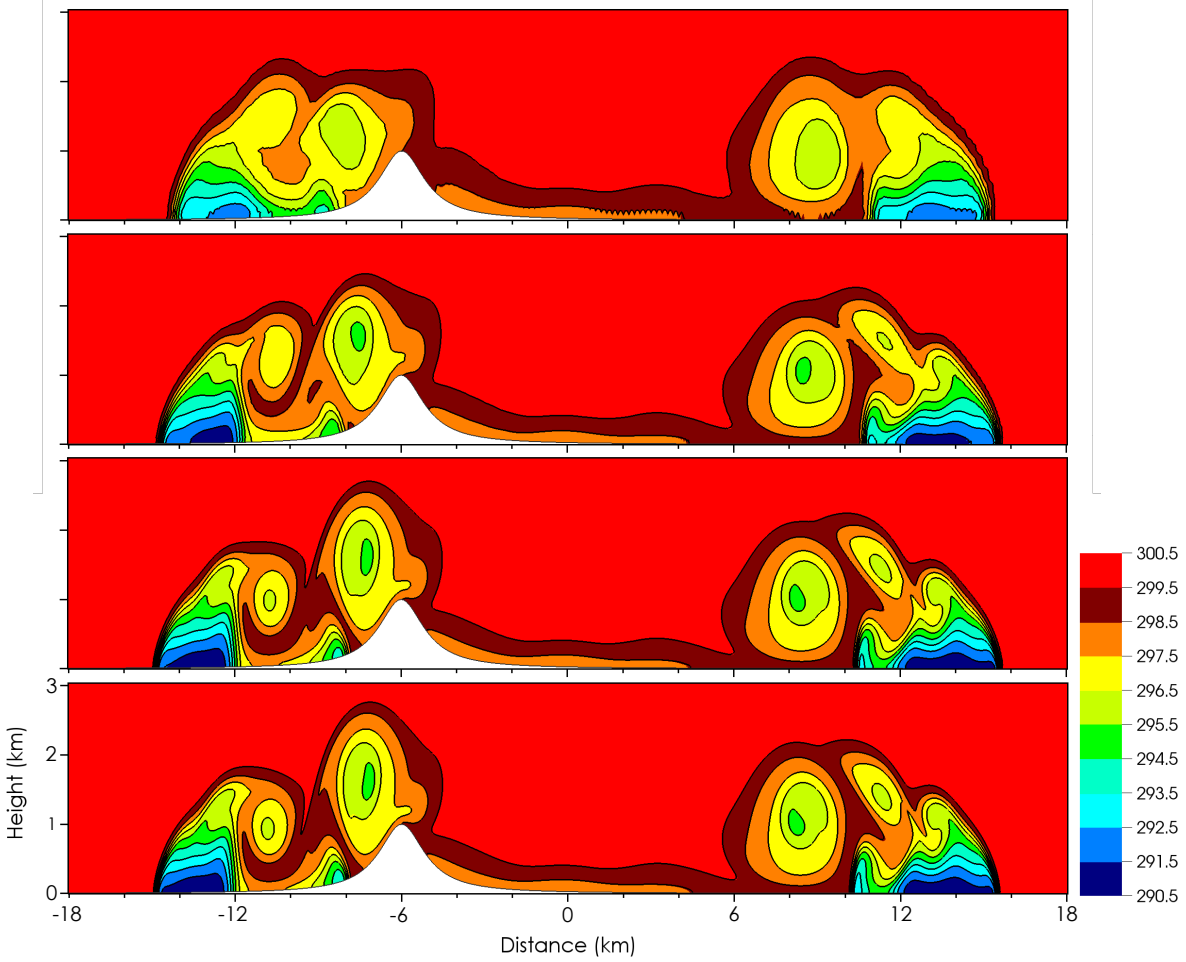

Figure 10. Potential temperature field at $t=900 \mathrm{~s}$ for the density current test case with an Agnesi hill on the left side of the domain and for different grid spacings $\Delta x=\Delta z=200,100,50$, and $25 \mathrm{~m}$ (top to bottom).

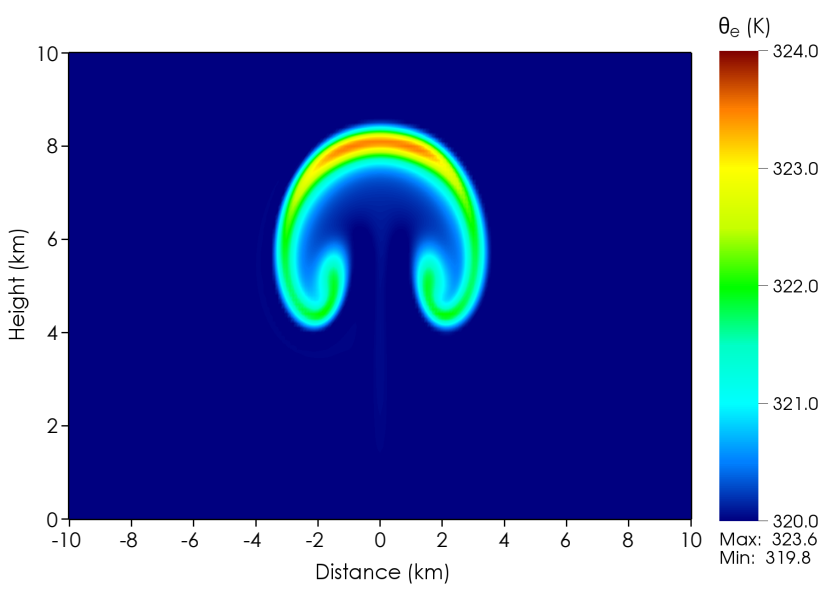

Figure 11. Equivalent potential temperature field for the moist rising bubble test case with background wind of $U=20 \mathrm{~m} \mathrm{~s}^{-1}$. Snapshot taken at $t=1000 \mathrm{~s}$ simulation time.

Figure 9 shows the temporal evolution of the total energy error for both simulations. In a dry atmosphere, the total energy is

$$
E_{\mathrm{d}}=\rho\left(q_{\mathrm{d}} c_{\mathrm{vd}} T+g z+0.5|\boldsymbol{v}|^{2}\right)
$$

Since exact energy conservation is not expected due to the model design, there is some kind of energy loss for both sim- ulations in the order of $10^{-3} \%$ at the end of the integration time. However, this is still acceptable due to the fact that in the test case there are very sharp gradients in potential temperature and wind speeds. Also, the difference of the total energy error between the two cases is very small $\left(10^{-4} \%\right)$. This means that, in this case, cut cells do not affect the conservation properties in the model at all. Total mass is always conserved within machine precision.

Another analysis is carried out by switching on the physical viscosity of $v=75 \mathrm{~m}^{2} \mathrm{~s}^{-1}$ as in Straka et al. (1993). Four simulations are performed with different isotropic grid spacings of $200,100,50$ and $25 \mathrm{~m}$, respectively. The potential temperature field after $900 \mathrm{~s}$ of integration time for these spatial resolutions is shown in Fig. 10. Table 4 shows minimum/maximum values of horizontal wind speed and potential temperature at this time. Skamarock et al. (2012) pointed out that their solutions show convergence at the $50 \mathrm{~m}$ spacing for this test case (without hill) with a fully compressible nonhydrostatic model. The same behavior can be observed with ASAM simulations (not shown here), which does also not change when the mountain is added to the domain. Despite that there is a slight change in maximum wind speed, the potential temperature field for the 25 and $50 \mathrm{~m}$ resolutions are nearly identical. Some notable differences in the field can be observed for the $100 \mathrm{~m}$ resolution, which are even more pronounced for the $200 \mathrm{~m}$ simulation. 
Table 4. Convergence study for the density current test case with a $1 \mathrm{~km}$ tall hill. Minimum/maximum values of horizontal velocity and potential temperature for different grid spacings.

\begin{tabular}{lcccc}
\hline$\Delta x$ & $u_{\min }\left(\mathrm{ms}^{-1}\right)$ & $u_{\max }\left(\mathrm{m} \mathrm{s}^{-1}\right)$ & $\theta_{\min }(\mathrm{K})$ & $\theta_{\max }(\mathrm{K})$ \\
\hline 200 & -25.93 & 35.64 & 291.89 & 300.01 \\
100 & -28.87 & 38.52 & 290.85 & 300.01 \\
50 & -28.90 & 38.31 & 290.71 & 300.00 \\
25 & -28.91 & 37.89 & 290.70 & 300.00 \\
\hline
\end{tabular}

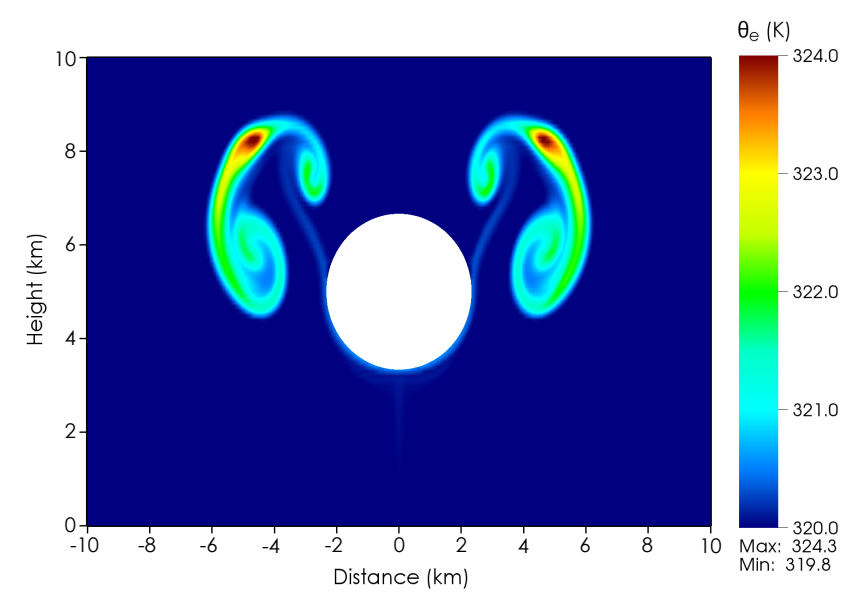

Figure 12. Equivalent potential temperature field for the moist rising bubble test including a zeppelin-shaped cut area in the center of the domain. Snapshot taken at $t=1250 \mathrm{~s}$ simulation time.

\subsection{Moist bubble with mid-air zeppelin}

The moist bubble benchmark case after Bryan and Fritsch (2002) is based on its dry counterpart described in Wicker and Skamarock (1998). There, a hydrostatic and neutrally balanced initial state is realized by a constant potential temperature. A warm perturbation in the center of the domain leads to the rising thermal. For the present test case, a moist neutral state can be expressed with the equivalent potential temperature $\theta_{\mathrm{e}}$ and two assumptions: the total water mixing ratio $r_{\mathrm{t}}=r_{\mathrm{v}}+r_{\mathrm{l}}$ remains constant and phase changes between water vapor and liquid water are exactly reversible. The perturbation field is identical to the dry bubble test case (Eqs. 75 and 76). The domain is $20 \mathrm{~km}$ long in the $x$ direction and the vertical extent is $10 \mathrm{~km}$. Grid spacing is again isotropic with $\Delta x=\Delta z=100 \mathrm{~m}$. Periodic boundary conditions are applied in the lateral direction, whereas free-slip conditions are used for the top and bottom boundaries. Again, a total energy test is performed by comparing two modifications of the present test case: (a) a uniform horizontal wind speed of $U=20 \mathrm{~m} \mathrm{~s}^{-1}$ is applied. With that, the center of the bubble is again located at $x=0 \mathrm{~m}$ at $t=1000 \mathrm{~s}$ after passing through the periodic boundaries. (b) In the center of the domain, a zeppelin-shaped region is cut out and acts as an obstacle for the rising bubble. A similar test like this was already intro-

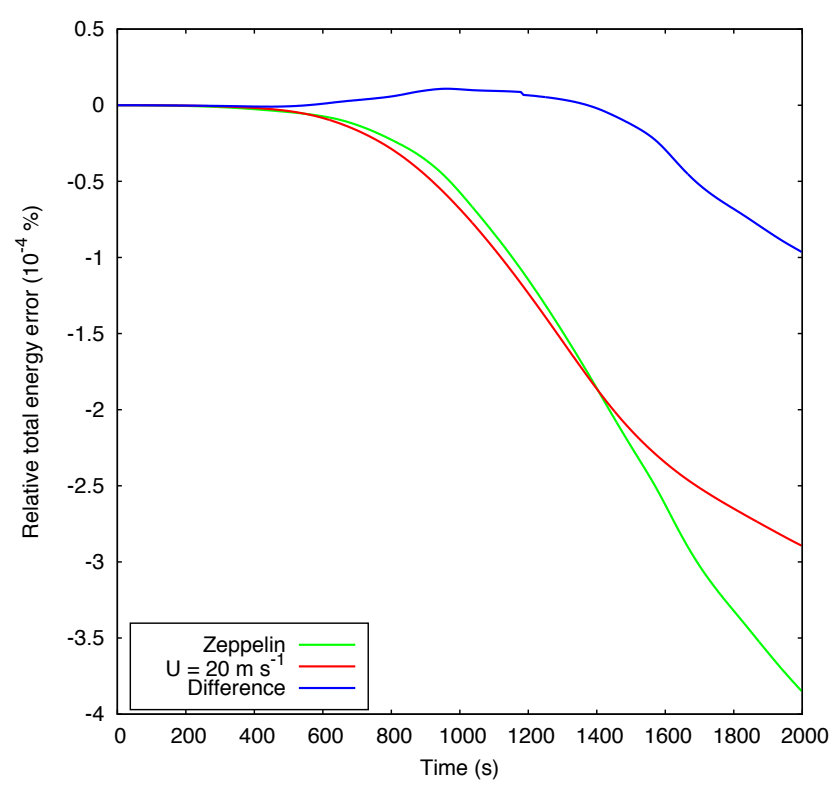

Figure 13. Same as in Fig. 9, but for the zeppelin and the lateral transported moist bubble test cases.

duced in Klein et al. (2009) and Jebens et al. (2011). However, their tests were carried out with the dry bubble, which was also shifted $1 \mathrm{~km}$ to the left. The result for the first case is shown in Fig. 11. The equivalent potential temperature field is very close to the benchmark simulation, despite that the maximum value of $\theta_{\mathrm{e}}$ is a little bit lower in our case compared to the literature values and that there is a slight asymmetry at the top of the thermal due to lateral transport. The position of the rising thermal for the zeppelin case after $t=1250 \mathrm{~s}$ is shown in Fig. 12. Because of the centered obstacle, the bubble is split into two parts and deformed but, still, two typical rotors are formed by each bubble and the result remains symmetric. When moisture and liquid water are present, the total energy takes the form

$E_{t}=\rho\left(\left[q_{\mathrm{d}} c_{\mathrm{vd}}+q_{\mathrm{v}} c_{\mathrm{vv}}+q_{1} c_{\mathrm{pl}}\right] T+q_{\mathrm{v}} L_{00}+g z+0.5|\boldsymbol{v}|^{2}\right)$.

Again, energy is not fully conserved but the total relative energy error after $2000 \mathrm{~s}$ of simulation time (there, in both cases, the bubbles reach the top boundary resulting in zonal divergence) stays in an acceptable range of $10^{-4} \%$ (Fig. 13), which is 1 order of magnitude smaller than in the cold bubble test case. The difference of the error in total energy between the zeppelin and the classical case is again very small. So, even with very small cut cells $(\approx 1 \%$ of full cell volume) and microphysical conversions there is no indication that conservation properties are deteriorated. For all cases, total mass is conserved within the numerical accuracy. After Bryan and Fritsch (2002), both mass and energy conservation are required to obtain the benchmark result. 
Table 5. Convergence study for the annulus advection test. $L_{1}$ error norm (full domain), experimental order of convergence (EOC), $L_{\infty}$ error norm, minimum and maximum tracer values for different meshes.

\begin{tabular}{lccccc}
\hline$N$ & Domain $L_{1}$ error & EOC & $L_{\infty}$ error & $\phi_{\min }$ & $\phi_{\max }$ \\
\hline 50 & $1.6377 \times 10^{-2}$ & - & $1.9176 \times 10^{-1}$ & $-2.0022 \times 10^{-3}$ & 1.00048 \\
100 & $4.9439 \times 10^{-3}$ & 1.73 & $1.1860 \times 10^{-1}$ & $-8.3884 \times 10^{-4}$ & 1.00066 \\
200 & $1.3653 \times 10^{-3}$ & 1.86 & $6.3112 \times 10^{-2}$ & $-8.5424 \times 10^{-13}$ & 0.99977 \\
400 & $3.7196 \times 10^{-4}$ & 1.88 & $3.1822 \times 10^{-2}$ & $-2.6209 \times 10^{-16}$ & 0.99977 \\
800 & $9.7302 \times 10^{-5}$ & 1.93 & $1.9877 \times 10^{-2}$ & $-1.0274 \times 10^{-12}$ & 0.99978 \\
\hline
\end{tabular}
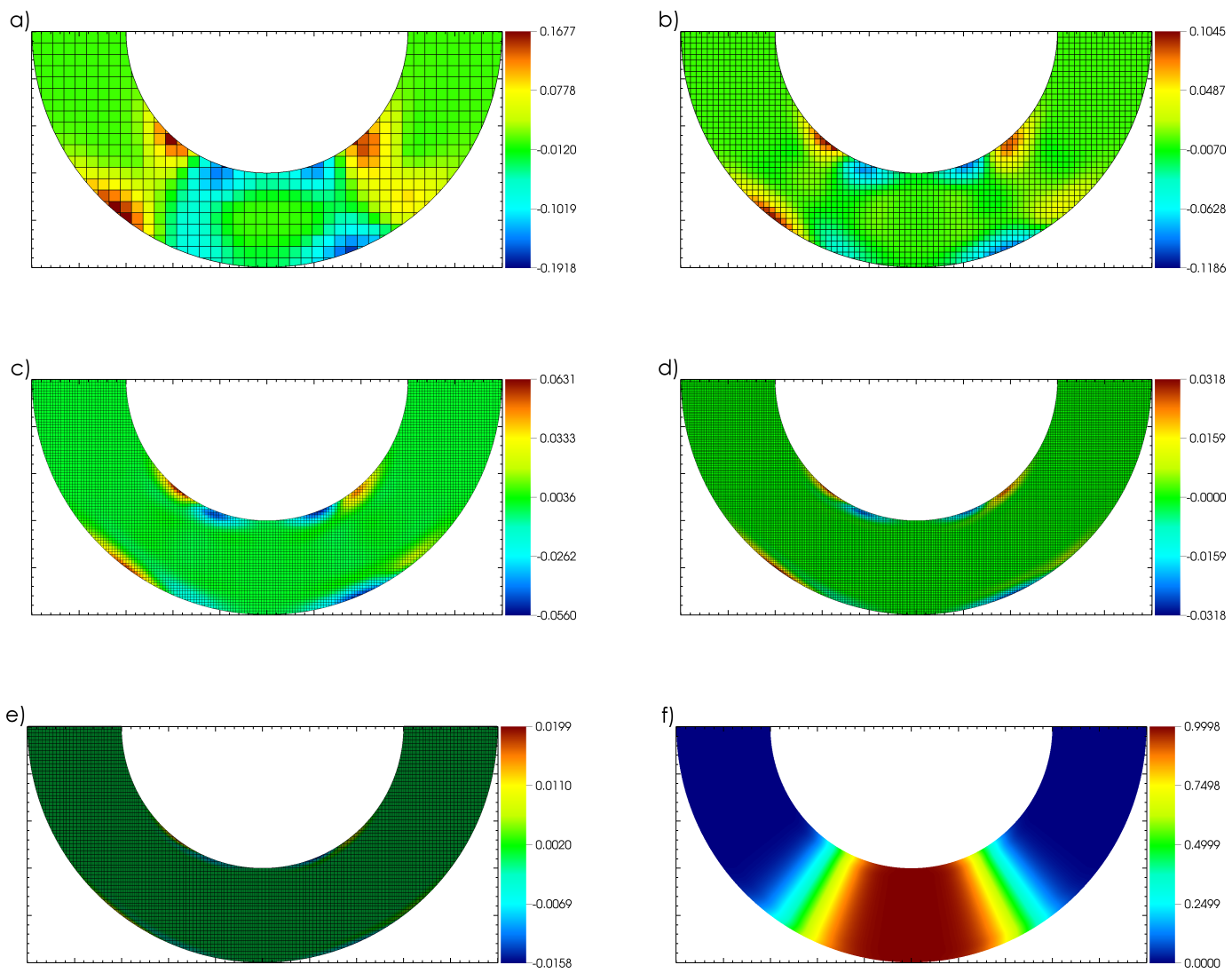

Figure 14. Computational meshes and difference scalar fields of $\phi$ for (a) $N=50$, (b) $N=100$, (c) $N=200$, (d) $N=400$, (e) $N=800$, and (f) the scalar field for $N=400$ after one rotation.

\subsection{Annulus advection test}

The test problem reported in Berger and Helzel (2012) describes the advection of a smooth bump by a radial wind field in an annulus. It is described by the radius of the inner circle $R_{1}=0.75$ and the radius of the outer circle $R_{2}=1.25$ within a rectangular domain $[-1.5,1.5] \times[-1.5,1.5]$. The initial scalar field takes the following form:

$\phi=0.5(\operatorname{erf}[5\{\vartheta-\pi / 3\}]+\operatorname{erf}[5\{2 \pi / 3-\vartheta\}])$,

where $\vartheta=\arctan (y / x)$. Deriving the velocity field from the stream function $\psi(x, y)=\pi\left(R_{2}^{2}-r^{2}\right) / 5$ with $r=\left(x^{2}+\right.$ $\left.y^{2}\right)^{1 / 2}$, one full rotation is reached at $t=5 \mathrm{~s}$. Figure $14 \mathrm{a}-\mathrm{e}$ shows the difference fields between the analytical and numerical solutions $(\Delta \phi)$ for different mesh sizes, where $N$ is the amount of grid cells in each spatial direction. Figure $14 \mathrm{f}$ shows the final field after $5 \mathrm{~s}$ of integration time for $N=400$. With greater $N$, the order of magnitude of the error decreases for the inner and outer boundaries and the intermediate part of the annulus is less affected. Table 5 shows the results of the convergence study, including error norms, experimental orders of convergence (EOC) as well as minimum and maximum values of the tracer field for different mesh sizes. For a fixed time step $(0.01,0.005,0.0025$ and $0.00125 \mathrm{~s}$, respectively) the advection scheme used in ASAM together with the Koren limiter shows almost second-order convergence in the 


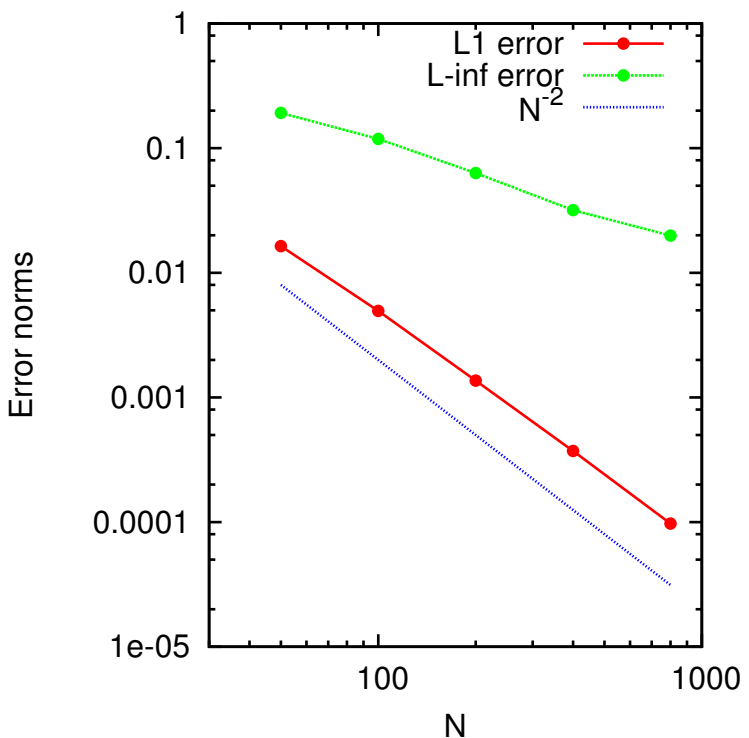

Figure 15. Convergence study for the annulus advection test. L1 (red) and $L_{\infty}$ (green) error norms for the full domain and reference line (blue dotted) for "perfect" second-order convergence.

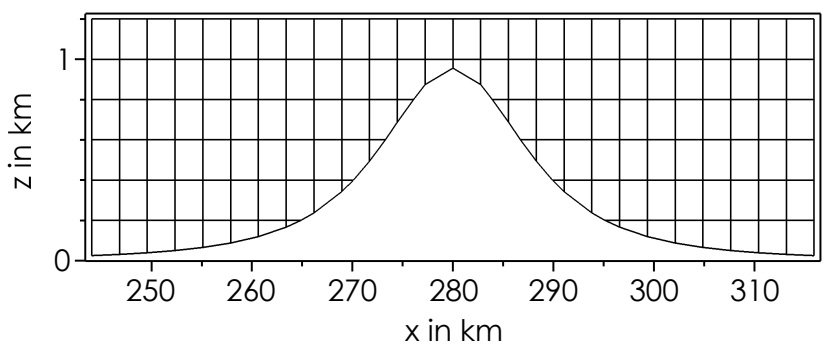

Figure 16. Computational grid around the mountain for an $x-z$ cut plane at $y=1.38 \mathrm{~km}$ (cell center).

$L_{1}$ norm, whereas the $L_{\infty}$ norm is nearly first-order accurate (see Fig. 15).

\subsection{3-D mountain flow in a moist atmosphere}

In this section, a test case described in Kunz and Wassermann (2011) is chosen. It includes forced lifting around a $1 \mathrm{~km}$ high mountain (see Fig. 16), latent heat release and orographic precipitation. Compared to the first three test cases, this case is now three-dimensional and uses a more realistic initial profile, which mimics atmospheric conditions when it comes to orographically dominated precipitation in the mountainous area of southwestern Germany. In their work, they used the three-dimensional, nonhydrostatic weather prediction model COSMO with terrain-following coordinates to describe the orography of the idealized mountain. The model setup for the ASAM simulations is as follows: the domain extends $553 \mathrm{~km} \times 553 \mathrm{~km}$ with a horizontal grid spacing of $2.765 \mathrm{~km}$ and 70 vertical layers with uniform spacing of $\Delta z=200 \mathrm{~m}$.

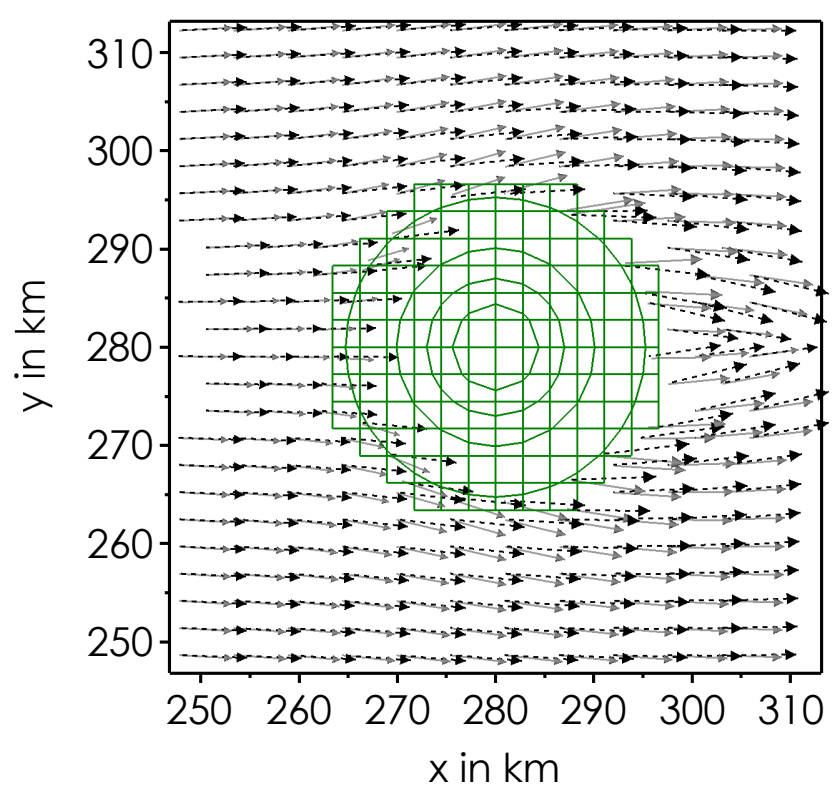

Figure 17. Horizontal cross section of horizontal wind vectors at $z=200 \mathrm{~m}$ height for the RH95 case (black) and the RH50 case (grey). Surface grid cells around the mountain in green, circle lines represent $200 \mathrm{~m}$ orography intervals.

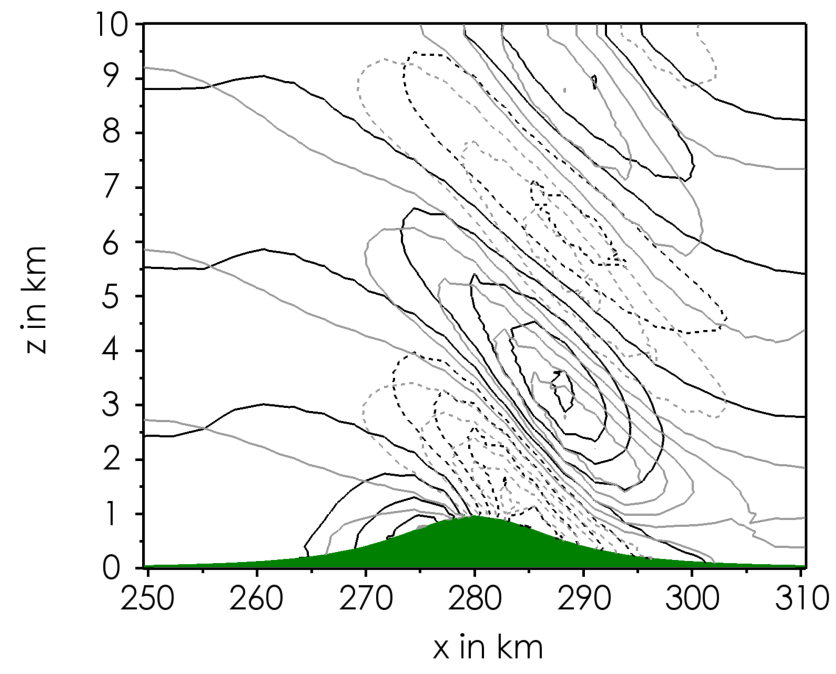

Figure 18. Vertical cross section ( $x-z$ plane) of vertical wind speed for the RH95 case (black) and the RH50 case (grey). Updrafts in solid lines $\left(0.2 \mathrm{~m} \mathrm{~s}^{-1}\right.$ contour interval, zero line included), downdrafts in dashed lines $\left(0.2 \mathrm{~ms}^{-1}\right.$ contour interval, zero line excluded).

A bell-shaped mountain is located at the center of the domain:

$h(x, y)=\frac{H}{\left(\frac{x^{2}+y^{2}}{a^{2}}+1\right)^{1.5}}$

with the mountain peak height $H=1 \mathrm{~km}$ and the half-width length $a=11 \mathrm{~km}$. Inflow and outflow boundary conditions 


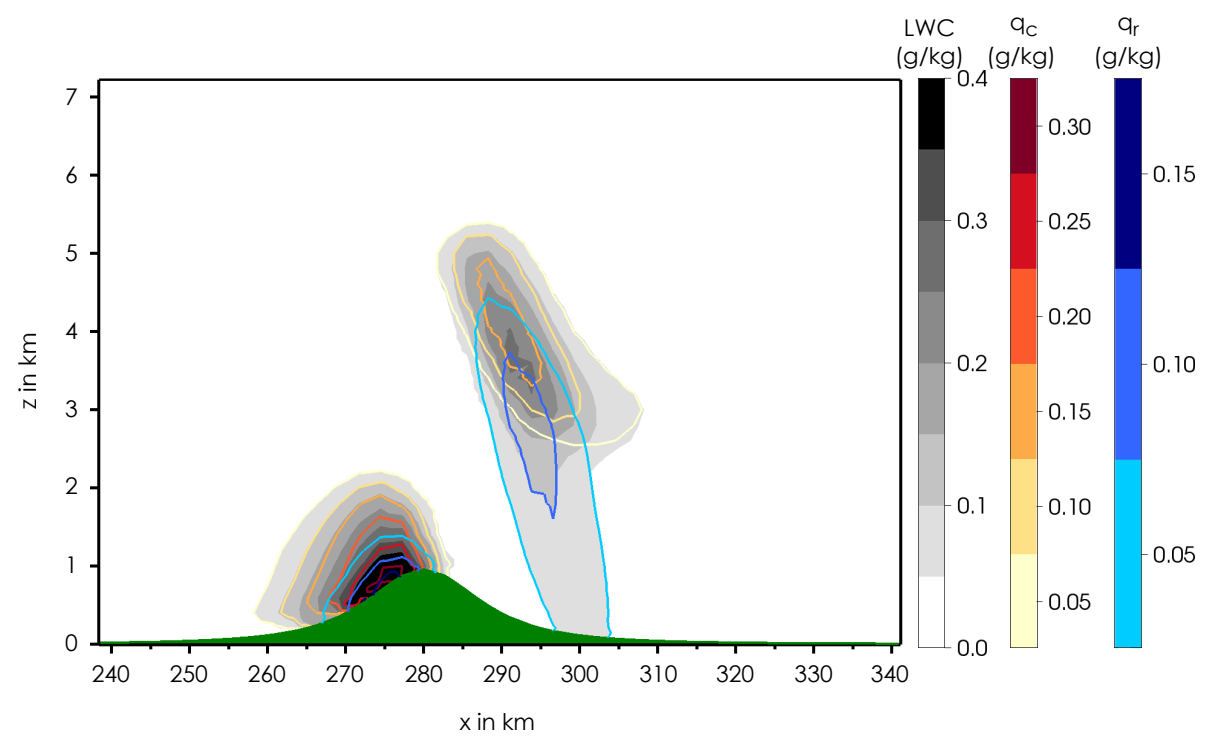

Figure 19. Vertical cross section ( $x-z$ plane) of microphysical properties for the RH95 case. Liquid water content (shaded), contours of specific cloud water content $q_{\mathrm{c}}$ (red-yellow) and specific rain water content $q_{\mathrm{r}}$ (blue).

are set according to the initial conditions. A Rayleigh damping layer above $11 \mathrm{~km}$ is applied to suppress gravity wave reflections from the top boundary. Surface heat fluxes and Coriolis force are turned off. For turbulence parameterization, the standard Smagorinsky subgrid-scale model is used. Microphysics are parameterized by the warm (i.e., no ice phase present) two-moment scheme described in Sect. 3.2. Initial profiles are obtained by assuming hydrostatic equilibrium, a near-surface temperature $T_{\mathrm{S}}=283.15 \mathrm{~K}$, a constant mean flow $U=10 \mathrm{~m} \mathrm{~s}^{-1}$, a constant dry static stability $N_{\mathrm{d}}=11 \times 10^{-3} \mathrm{~s}^{-1}$ and a relative humidity profile, which is constant up to $z_{\mathrm{m}}=5 \mathrm{~km}$ and rapidly decreases above this level according to

$\mathrm{RH}(z)=\mathrm{RH}_{\mathrm{S}}\left[0.5+\pi^{-1} \arctan \left(\frac{z-z_{\mathrm{m}}}{500}\right)\right]$

with the near-surface humidity $\mathrm{RH}_{\mathrm{S}}=95 \%$ (RH95 case). To compare the results with its dry counterpart, another simulation with $\mathrm{RH}_{\mathrm{S}}=50 \%$ is performed (RH50 case). Figure 17 shows the wind field at $200 \mathrm{~m}$ height around the mountain for both cases. In the nearly saturated atmosphere, there is a more direct overflow over the mountain, which is caused by the reduced stability due to high moisture. These different flow characteristics also affect gravity wave structure (Fig. 18). The resulting waves are steeper and have a greater wavelength, which is in agreement with gravity wave theory and the results from Kunz and Wassermann (2011). Most notable differences in the numerical results are discrepancies in vertical wind strength in the lowest model layer at the windward side the mountain $\left(w \approx 0.6 \mathrm{~m} \mathrm{~s}^{-1}\right.$ in ASAM vs.

$w \approx 0.2 \mathrm{~m} \mathrm{~s}^{-1}$ in COSMO), which can be explained by the different surface coordinate systems of the models (Cartesian grid with cut cells in ASAM and generalized terrain- following coordinates in COSMO). Overall, the amplitude of vertical wind is higher for the resulting gravity waves. Typical patterns of orographic clouds (one cloud upstream of the mountain and a larger cloud with a high amount of liquid water content (LWC) and precipitation that reaches the ground in the lee of the mountain) are also reproduced (Fig. 19). In this particular case the resulting patterns as well as the cloud and rain water contents are comparable to the literature results, despite using different coordinate systems and cloud microphysical schemes.

\section{Conclusions and future work}

A detailed description of the three-dimensional, fully compressible, nonhydrostatic All Scale Atmospheric Model (ASAM) was presented. Main focus of this work was the description of the cut cell method within a Cartesian grid structure. With this method there is no accuracy loss near steep slopes, which can occur around mountains using a high spatial resolution or when obstacles or buildings are embedded. The concepts of the spatial discretization of the advection operator and a nonlinear term in the momentum equation were outlined. A technique to distribute surface fluxes around cut cells was described. An implicit Rosenbrock time integration scheme with two splitting approaches of the Jacobian were presented, which is particularly useful to bypass the small cell problem. With the described scheme, relatively large time steps are possible. Physical parameterizations (Smagorinsky subgrid-scale model, two-moment warm microphysics scheme, multi-layer soil model) which are necessary for performing particular atmospheric simulations, e.g., large eddy simulations of marine boundary layers, are 
implemented in ASAM. The model produces good results when comparing scalar and velocity fields for typical benchmark test cases from the literature. It was shown that energy conservation is not affected when it comes to interaction with the flow in the vicinity of cut cells. However, perfect energy conservation cannot be expected by design. Accuracy tests show that the EOC is almost second-order for the annulus advection test.

Other model features that could not be presented in the framework of this paper are local mesh refinement and parallel usage of the model. They will be part of future studies. There, performance tests for highly parallel computing with a large number of processors will be conducted. Furthermore, high-frequency output is desired for statistical data analysis. For this reason, efficient techniques like adaption of the output on modern parallel visualization software will be developed. There are no operator splitting techniques used, which leads to a consistent treatment of new processes with respect to time and to a simple programming style for the most part.
Another focus on future model development lies in the model physics, which includes further testing of current implementations as well as adding new parameterizations, e.g., an ice microphysics scheme. For the description of turbulence, other (dynamic) Smagorinsky models (e.g., Kleissl et al., 2006; Porté-Agel et al., 2000) might be better suited for particular simulations compared to the present model version. Also, a so-called implicit LES will be tested and verified. There, no turbulence model is used and the numerics of the discretization generate unresolved turbulent motions themselves. In this type of LES, the sensitivity of the thermodynamical formulation (especially in the energy equation) on the resulting motions has to be analyzed.

ASAM already was and will further be applied for large eddy simulations of urban and marine boundary layers. Another ongoing study deals with island effects on boundary layer modification in the trade wind area exemplified by the island of Barbados, where the island topography plays a significant role and can be well described by the cut cell method. 


\section{Appendix A: Derivation of tendency equations}

In this section, a straightforward derivation of the density potential temperature tendency equation is given to get the necessary source terms for microphysics, surface fluxes and precipitation. Therefore, phase changes are allowed and a water vapor source term $S_{\mathrm{v}}$ and sedimentation velocity $W_{\mathrm{f}}$ for rain drops are added to the system.

$\frac{\mathrm{d} \rho}{\mathrm{d} t}=-\rho \nabla \cdot \boldsymbol{v}+S_{\mathrm{v}}-S_{\text {fall }}$,

$\frac{\mathrm{d} \rho_{\mathrm{v}}}{\mathrm{d} t}=-\rho_{\mathrm{v}} \nabla \cdot \boldsymbol{v}+S_{\mathrm{v}}+S_{\mathrm{ph}}$

$\frac{\mathrm{d} \rho_{\mathrm{l}}}{\mathrm{d} t}=-\rho_{\mathrm{c}} \nabla \cdot \boldsymbol{v}-S_{\mathrm{ph}}-S_{\text {fall }}$,

$\frac{\mathrm{d} \rho_{\mathrm{d}}}{\mathrm{d} t}=-\rho_{\mathrm{d}} \nabla \cdot \boldsymbol{v}$.

The precipitation term is $S_{\text {fall }}=\partial / \partial z\left(\rho_{\mathrm{r}} W_{\mathrm{f}}\right)$ with the sedimentation velocity $W_{\mathrm{f}}$ after Eq. (56). One can rewrite Eqs. (A2) and (A3) with the mixing ratios $r_{\mathrm{v}}=\rho_{\mathrm{v}} / \rho_{\mathrm{d}}$ and $r_{1}=\rho_{1} / \rho_{\mathrm{d}}$ :

$\frac{\mathrm{d} r_{\mathrm{v}}}{\mathrm{d} t}=\frac{1}{\rho_{\mathrm{d}}}\left(S_{\mathrm{v}}+S_{\mathrm{ph}}\right)$,

$\frac{\mathrm{d} r_{1}}{\mathrm{~d} t}=-\frac{1}{\rho_{\mathrm{d}}}\left(S_{\mathrm{ph}}+S_{\text {fall }}\right)$.

For the sake of simplicity (regarding the following derivations) the liquid water density and mixing ratio are used with $\rho_{\mathrm{l}}=\rho_{\mathrm{c}}+\rho_{\mathrm{r}}$ or $r_{\mathrm{l}}=r_{\mathrm{c}}+r_{\mathrm{r}}$. The model however solves the prognostic equations for the cloud water density $\rho_{\mathrm{c}}$ and rain water density $\rho_{\mathrm{r}}$ separately.

\section{A1 Internal energy and absolute temperature}

A prognostic equation for the internal energy $e$ is derived from the first law of thermodynamics; cf. Bott (2008, Eq. 31) and Satoh et al. (2008, Eq. B.13),

$\frac{\partial(\rho e)}{\partial t}+\nabla \cdot(\rho e \boldsymbol{v})=-p \nabla \cdot \boldsymbol{v}+S_{e}-\frac{\partial}{\partial z}\left(\rho_{\mathrm{r}} W_{\mathrm{f}} e_{1}\right)-\rho_{\mathrm{r}} W_{\mathrm{f}} g$,

and alternatively with the specific enthalpy $h$ in the advection part,

$$
\frac{\partial(\rho e)}{\partial t}+\nabla \cdot(\rho h \boldsymbol{v})=\boldsymbol{v} \cdot \nabla p+S_{e}-\frac{\partial}{\partial z}\left(\rho_{\mathrm{r}} W_{\mathrm{f}} e_{1}\right)-\rho_{\mathrm{r}} W_{\mathrm{f}} g .
$$

There, the total specific internal energy is

$e=h-\frac{p}{\rho}=\left(q_{\mathrm{d}} c_{\mathrm{vd}}+q_{\mathrm{v}} c_{\mathrm{vv}}+q_{1} c_{\mathrm{pl}}\right) T+q_{\mathrm{v}} L_{00}$,

and the specific internal energy for liquid water

$e_{1}=h_{1}=c_{\mathrm{pl}} T$.
The term $S_{e}$ is related to the water vapor source term:

$S_{e}=h_{\mathrm{v}} S_{\mathrm{v}}$.

Transforming Eq. (A7) into a tendency equation for the absolute temperature:

$$
\begin{aligned}
& e\left(\frac{\partial \rho}{\partial t}+\nabla \cdot(\rho v)\right)+\rho\left(\frac{\partial e}{\partial t}+v \cdot \nabla e\right)=\operatorname{RHS}(\mathrm{A} 7), \\
& e\left(S_{\mathrm{v}}-S_{\mathrm{fall}}\right)+\rho \frac{\mathrm{d} e}{\mathrm{~d} t}=\operatorname{RHS}(\mathrm{A} 7) \\
& e\left(S_{\mathrm{v}}-S_{\mathrm{fall}}\right)+\frac{\mathrm{d}(\rho e)}{\mathrm{d} t}-e \frac{\mathrm{d} \rho}{\mathrm{d} t}=\operatorname{RHS}(\mathrm{A} 7) .
\end{aligned}
$$

With Eq. (A9), this leads to

$$
\begin{aligned}
\frac{\mathrm{d}(\rho e)}{\mathrm{d} t}= & \frac{\mathrm{d}}{\mathrm{d} t}\left(\left[\rho_{\mathrm{d}} c_{\mathrm{vd}}+\rho_{\mathrm{v}} c_{\mathrm{vv}}+\rho_{\mathrm{l}} c_{\mathrm{pl}}\right] T+\rho_{\mathrm{v}} L_{00}\right) \\
= & T\left(c_{\mathrm{vd}} \frac{\mathrm{d} \rho_{\mathrm{d}}}{\mathrm{d} t}+c_{\mathrm{vv}} \frac{\mathrm{d} \rho_{\mathrm{v}}}{\mathrm{d} t}+c_{\mathrm{pl}} \frac{\mathrm{d} \rho_{1}}{\mathrm{~d} t}\right) \\
& +\left(\rho_{\mathrm{d}} c_{\mathrm{vd}}+\rho_{\mathrm{v}} c_{\mathrm{vv}}+\rho_{\mathrm{l}} c_{\mathrm{pl}}\right) \frac{\mathrm{d} T}{\mathrm{~d} t}+L_{00} \frac{\mathrm{d} \rho_{\mathrm{v}}}{\mathrm{d} t} \\
& -\left(\rho_{\mathrm{d}} c_{\mathrm{vd}}+\rho_{\mathrm{v}} c_{\mathrm{vv}}+\rho_{\mathrm{l}} c_{\mathrm{pl}}\right) T \nabla \cdot \boldsymbol{v} \\
& +T c_{\mathrm{vv}}\left(S_{\mathrm{v}}+S_{\mathrm{ph}}\right)-T c_{\mathrm{pl}}\left(S_{\mathrm{ph}}+S_{\mathrm{fall}}\right) \\
& +\left(\rho_{\mathrm{d}} c_{\mathrm{vd}}+\rho_{\mathrm{v}} c_{\mathrm{vv}}+\rho_{1} c_{\mathrm{pl}}\right) \frac{\mathrm{d} T}{\mathrm{~d} t} \\
& -L_{00}\left(\rho_{\mathrm{v}} \nabla \cdot \boldsymbol{v}-S_{\mathrm{v}}-S_{\mathrm{ph}}\right) \\
= & -e \rho \nabla \cdot \boldsymbol{v}+\left(\rho_{\mathrm{d}} c_{\mathrm{vd}}+\rho_{\mathrm{v}} c_{\mathrm{vv}}+\rho_{\mathrm{l}} c_{\mathrm{pl}}\right) \frac{\mathrm{d} T}{\mathrm{~d} t} \\
& +T\left(c_{\mathrm{vv}}\left[S_{\mathrm{v}}+S_{\mathrm{ph}}\right]-c_{\mathrm{pl}}\left[S_{\mathrm{ph}}+S_{\mathrm{fall}}\right]\right) \\
& +L_{00}\left(S_{\mathrm{v}}+S_{\mathrm{ph}}\right), \\
e \frac{\mathrm{d} \rho}{\mathrm{d} t}= & e\left(S_{\mathrm{v}}-S_{\mathrm{fall}}\right)-e \rho \nabla \cdot \boldsymbol{v} .
\end{aligned}
$$

Inserting Eqs. (A13) and (A14) in Eq. (A12):

$$
\begin{aligned}
\rho_{\mathrm{d}} c_{\mathrm{vml}} \frac{\mathrm{d} T}{\mathrm{~d} t}= & -T\left(c_{\mathrm{vv}}\left[S_{\mathrm{v}}+S_{\mathrm{ph}}\right]-c_{\mathrm{pl}}\left[S_{\mathrm{ph}}+S_{\mathrm{fall}}\right]\right) \\
& -L_{00}\left(S_{\mathrm{v}}+S_{\mathrm{ph}}\right)-p \nabla \cdot \boldsymbol{v}+S_{\mathrm{v}} h_{\mathrm{v}} \\
& -\frac{\partial}{\partial z}\left(\rho_{\mathrm{r}} W_{\mathrm{f}} e_{1}\right)-\rho_{\mathrm{r}} W_{\mathrm{f}} g \\
= & -p \nabla \cdot \boldsymbol{v}+\left(h_{\mathrm{v}}-c_{\mathrm{vv}} T-L_{00}\right) S_{\mathrm{v}} \\
& +\left(c_{\mathrm{pl}} T-c_{\mathrm{vv}} T-L_{00}\right) S_{\mathrm{ph}} \\
& -\frac{\partial}{\partial z}\left(\rho_{\mathrm{r}} W_{\mathrm{f}} e_{1}\right)-\rho_{\mathrm{r}} W_{\mathrm{f}} g .
\end{aligned}
$$

Here we define

$c_{\mathrm{vml}} \equiv c_{\mathrm{vd}}+r_{\mathrm{v}} c_{\mathrm{vv}}+r_{1} c_{\mathrm{pl}}$.

Rewriting the pressure and elimination of the velocity divergence:

$$
-p \nabla \cdot \boldsymbol{v}=-\left(\rho_{\mathrm{d}} R_{\mathrm{d}}+\rho_{\mathrm{v}} R_{\mathrm{v}}\right) T\left(-\frac{1}{\rho_{\mathrm{d}}} \frac{\mathrm{d} \rho_{\mathrm{d}}}{\mathrm{d} t}\right)
$$




$$
\begin{aligned}
= & \left(R_{\mathrm{d}}+r_{\mathrm{v}} R_{\mathrm{v}}\right) T \frac{\mathrm{d} \rho_{\mathrm{d}}}{\mathrm{d} t}, \\
\frac{\mathrm{d} \rho_{\mathrm{d}}}{\mathrm{d} t}= & \frac{1}{R_{\mathrm{d}} T\left(1+\frac{r_{\mathrm{v}}}{\varepsilon}\right)} \frac{\mathrm{d} p}{\mathrm{~d} t}-\frac{p R_{\mathrm{d}} T}{\varepsilon\left(R_{\mathrm{d}} T\left[1+\frac{r_{\mathrm{v}}}{\varepsilon}\right]\right)^{2}} \frac{\mathrm{d} r_{\mathrm{v}}}{\mathrm{d} t} \\
& -\frac{p}{R_{\mathrm{d}} T^{2}\left(1+\frac{r_{\mathrm{v}}}{\varepsilon}\right)} \frac{\mathrm{d} T}{\mathrm{~d} t} \\
= & \frac{\rho_{\mathrm{d}}}{p} \frac{\mathrm{d} p}{\mathrm{~d} t}-\frac{\rho_{\mathrm{d}}}{\varepsilon+r_{\mathrm{v}}} \frac{\mathrm{d} r_{\mathrm{v}}}{\mathrm{d} t}-\frac{\rho_{\mathrm{d}}}{T} \frac{\mathrm{d} T}{\mathrm{~d} t} \\
\Rightarrow-p \nabla \cdot \boldsymbol{v}= & \frac{\mathrm{d} p}{\mathrm{~d} t}-\rho_{\mathrm{d}} R_{\mathrm{v}} T \frac{\mathrm{d} r_{\mathrm{v}}}{\mathrm{d} t}-\left(\rho_{\mathrm{d}} R_{\mathrm{d}}+\rho_{\mathrm{v}} R_{\mathrm{v}}\right) \frac{\mathrm{d} T}{\mathrm{~d} t} \\
= & \frac{\mathrm{d} p}{\mathrm{~d} t}-R_{\mathrm{v}} T\left(S_{\mathrm{v}}+S_{\mathrm{ph}}\right)-\left(\rho_{\mathrm{d}} R_{\mathrm{d}}+\rho_{\mathrm{v}} R_{\mathrm{v}}\right) \frac{\mathrm{d} T}{\mathrm{~d} t} .
\end{aligned}
$$

This now leads to the temperature equation:

$$
\begin{aligned}
\rho_{\mathrm{d}}\left(c_{\mathrm{vml}}+R_{\mathrm{m}}\right) \frac{\mathrm{d} T}{\mathrm{~d} t}= & \rho_{\mathrm{d}} c_{\mathrm{pml}} \frac{\mathrm{d} T}{\mathrm{~d} t} \\
= & \frac{\mathrm{d} p}{\mathrm{~d} t}-R_{\mathrm{v}} T\left(S_{\mathrm{v}}+S_{\mathrm{ph}}\right) \\
& +\left(h_{\mathrm{v}}-c_{\mathrm{vv}} T-L_{00}\right) S_{\mathrm{v}} \\
& +\left(c_{\mathrm{pl}} T-c_{\mathrm{vv}} T-L_{00}\right) S_{\mathrm{ph}} \\
& -\frac{\partial}{\partial z}\left(\rho_{\mathrm{r}} W_{\mathrm{f}} e_{1}\right)-\rho_{\mathrm{r}} W_{\mathrm{f}} g .
\end{aligned}
$$

With $c_{\mathrm{pv}}-c_{\mathrm{vv}}=R_{\mathrm{v}}$, the water vapor source term disappears:

$$
\begin{aligned}
& \left(h_{\mathrm{v}}-c_{\mathrm{vv}} T-L_{00}-R_{\mathrm{v}} T\right) S_{\mathrm{v}} \\
& \quad=\left(c_{\mathrm{pv}} T+L_{00}-c_{\mathrm{vv}} T-L_{00}-R_{\mathrm{v}} T\right) S_{\mathrm{v}}=0 .
\end{aligned}
$$

Further simplifying,

$$
\begin{aligned}
& \left(c_{\mathrm{pl}} T-c_{\mathrm{vv}} T-R_{\mathrm{v}} T-L_{00}\right) S_{\mathrm{ph}} \\
& \quad=\left(c_{\mathrm{pl}} T-c_{\mathrm{vv}} T-R_{\mathrm{v}} T-L_{\mathrm{v}}+\left(c_{\mathrm{pv}}-c_{\mathrm{pl}}\right) T\right) S_{\mathrm{ph}} \\
& \quad=-L_{\mathrm{v}} S_{\mathrm{ph}} .
\end{aligned}
$$

Rearranging finally leads to the temperature equation,

$\rho_{\mathrm{d}} c_{\mathrm{pml}} \frac{\mathrm{d} T}{\mathrm{~d} t}=\frac{\mathrm{d} p}{\mathrm{~d} t}-L_{\mathrm{v}} S_{\mathrm{ph}}-\frac{\partial}{\partial z}\left(\rho_{\mathrm{r}} W_{\mathrm{f}} e_{1}\right)-\rho_{\mathrm{r}} W_{\mathrm{f}} g$,

and its logarithmic derivative

$$
\begin{aligned}
\frac{\mathrm{d} \ln T}{\mathrm{~d} t}= & \frac{R_{\mathrm{m}}}{c_{\mathrm{pml}}} \frac{\mathrm{d} \ln p}{\mathrm{~d} t}-\frac{L_{\mathrm{v}}}{\rho_{\mathrm{d}} c_{\mathrm{pml}} T} S_{\mathrm{ph}} \\
& -\frac{1}{\rho_{\mathrm{d}} c_{\mathrm{pml}} T} \frac{\partial}{\partial z}\left(\rho_{\mathrm{r}} W_{\mathrm{f}} e_{1}\right)-\frac{\rho_{\mathrm{r}} W_{\mathrm{f}} g}{\rho_{\mathrm{d}} c_{\mathrm{pml}} T} .
\end{aligned}
$$

\section{A2 Potential temperature}

A prognostic equation for the (moist) potential temperature is derived here. This is necessary because it appears in the density potential temperature equation later on. Quantities that contain water vapor and liquid water are marked with a tilde to distinguish them from their dry equivalents (e.g., dry potential temperature $\theta$ ).
The moist potential temperature is

$\tilde{\theta}=\frac{T}{\widetilde{\pi}} \quad$ with $\quad \tilde{\pi}=\left(\frac{p}{p_{0}}\right)^{\frac{R_{\mathrm{m}}}{c_{\mathrm{pml}}}}$.

Taking the logarithm of the Exner function $\tilde{\pi}$ leads to

$\ln \tilde{\pi}=\frac{R_{\mathrm{m}}}{c_{\mathrm{pml}}} \ln \left(\frac{p}{p_{0}}\right)$.

The time derivative is

$$
\begin{aligned}
\frac{\mathrm{d} \ln \tilde{\pi}}{\mathrm{d} t}= & \frac{R_{\mathrm{v}}}{R_{\mathrm{m}}} \ln \tilde{\pi} \frac{\mathrm{d} r_{\mathrm{v}}}{\mathrm{d} t}-\frac{\ln \tilde{\pi}}{c_{\mathrm{pml}}}\left(c_{\mathrm{pv}} \frac{\mathrm{d} r_{\mathrm{v}}}{\mathrm{d} t}+c_{\mathrm{pl}} \frac{\mathrm{d} r_{1}}{\mathrm{~d} t}\right) \\
& +\frac{R_{\mathrm{m}}}{c_{\mathrm{pml}}} \frac{\mathrm{d} \ln p}{\mathrm{~d} t} \\
= & \frac{R_{\mathrm{v}}}{R_{\mathrm{m}}} \ln \tilde{\pi} \frac{S_{\mathrm{v}}+S_{\mathrm{ph}}}{\rho_{\mathrm{d}}} \\
& -\ln \tilde{\pi}\left(\frac{c_{\mathrm{pv}}}{c_{\mathrm{pml}}} \frac{S_{\mathrm{v}}+S_{\mathrm{ph}}}{\rho_{\mathrm{d}}}-\frac{c_{\mathrm{pl}}}{c_{\mathrm{pml}}} \frac{S_{\mathrm{ph}}+S_{\mathrm{fall}}}{\rho_{\mathrm{d}}}\right) \\
& +\frac{R_{\mathrm{m}}}{c_{\mathrm{pml}}} \frac{\mathrm{d} \ln p}{\mathrm{~d} t} \\
= & \ln \tilde{\pi}\left(\left[\frac{R_{\mathrm{v}}}{R_{\mathrm{m}}}-\frac{c_{\mathrm{pv}}}{c_{\mathrm{pml}}}\right] \frac{S_{\mathrm{v}}+S_{\mathrm{ph}}}{\rho_{\mathrm{d}}}+\frac{c_{\mathrm{pl}}}{c_{\mathrm{pml}}} \frac{S_{\mathrm{ph}}+S_{\mathrm{fall}}}{\rho_{\mathrm{d}}}\right) \\
& +\frac{R_{\mathrm{m}}}{c_{\mathrm{pml}}} \frac{\mathrm{d} \ln p}{\mathrm{~d} t} \\
= & \frac{\ln \tilde{\pi}}{\rho_{\mathrm{d}}}\left(\frac{R_{\mathrm{v}}}{R_{\mathrm{m}}}-\frac{c_{\mathrm{pv}}}{c_{\mathrm{pml}}}\right) S_{\mathrm{v}}+\frac{\ln \tilde{\pi}}{\rho_{\mathrm{d}}}\left(\frac{R_{\mathrm{v}}}{R_{\mathrm{m}}}+\frac{c_{\mathrm{pl}}-c_{\mathrm{pv}}}{c_{\mathrm{pml}}}\right) S_{\mathrm{ph}} \\
& +\frac{\ln \tilde{\pi}}{\rho_{\mathrm{d}}} \frac{c_{\mathrm{pl}}}{c_{\mathrm{pml}}} S_{\mathrm{fall}}+\frac{R_{\mathrm{m}}}{c_{\mathrm{pml}}} \frac{\mathrm{d} \ln p}{\mathrm{~d} t},
\end{aligned}
$$

which leads us to the moist potential temperature equation:

$$
\begin{aligned}
\frac{\mathrm{d} \ln \tilde{\theta}}{\mathrm{d} t}= & \frac{\mathrm{d} \ln T}{\mathrm{~d} t}-\frac{\mathrm{d} \ln \tilde{\pi}}{\mathrm{d} t} \\
= & \frac{R_{\mathrm{m}}}{c_{\mathrm{pml}}} \frac{\mathrm{d} \ln p}{\mathrm{~d} t}-\frac{L_{\mathrm{v}}}{\rho_{\mathrm{d}} c_{\mathrm{pml}} T} S_{\mathrm{ph}}-\frac{1}{\rho_{\mathrm{d}} c_{\mathrm{pml}} T} \frac{\partial}{\partial z}\left(\rho_{\mathrm{r}} W_{\mathrm{f}} e_{1}\right) \\
& -\frac{\rho_{\mathrm{r}} W_{\mathrm{f}} g}{\rho_{\mathrm{d}} c_{\mathrm{pml}} T}-\frac{\ln \tilde{\pi}}{\rho_{\mathrm{d}}}\left(\frac{R_{\mathrm{v}}}{R_{\mathrm{m}}}-\frac{c_{\mathrm{pv}}}{c_{\mathrm{pml}}}\right) S_{\mathrm{v}} \\
& -\frac{\ln \tilde{\pi}}{\rho_{\mathrm{d}}}\left(\frac{R_{\mathrm{v}}}{R_{\mathrm{m}}}+\frac{c_{\mathrm{pl}}-c_{\mathrm{pv}}}{c_{\mathrm{pml}}}\right) S_{\mathrm{ph}}-\frac{\ln \tilde{\pi}}{\rho_{\mathrm{d}}} \frac{c_{\mathrm{pl}}}{c_{\mathrm{pml}}} S_{\mathrm{fall}} \\
& -\frac{R_{\mathrm{m}}}{c_{\mathrm{pml}}} \frac{\mathrm{d} \ln p}{\mathrm{~d} t} \\
= & -\frac{\ln \tilde{\pi}}{\rho_{\mathrm{d}}}\left(\frac{R_{\mathrm{v}}}{R_{\mathrm{m}}}-\frac{c_{\mathrm{pv}}}{c_{\mathrm{pml}}}\right) S_{\mathrm{v}} \\
& -\frac{1}{\rho_{\mathrm{d}}}\left(\frac{L_{\mathrm{v}}}{c_{\mathrm{pml}} T}+\ln \tilde{\pi}\left[\frac{R_{\mathrm{v}}}{R_{\mathrm{m}}}+\frac{c_{\mathrm{pl}}-c_{\mathrm{pv}}}{c_{\mathrm{pml}}}\right]\right) S_{\mathrm{ph}} \\
& -\frac{\ln \tilde{\pi}}{\rho_{\mathrm{d}}} \frac{c_{\mathrm{pl}}}{c_{\mathrm{pml}}} S_{\mathrm{fall}}-\frac{1}{\rho_{\mathrm{d}} c_{\mathrm{pml}} T} \frac{\partial}{\partial z}\left(\rho_{\mathrm{r}} W_{\mathrm{f}} e_{1}\right)-\frac{\rho_{\mathrm{r}} W_{\mathrm{f}} g}{\rho_{\mathrm{d}} c_{\mathrm{pml}} T} .
\end{aligned}
$$




\section{A3 Density potential temperature}

With the definition of the density potential temperature

$\theta_{\rho}=\tilde{\theta} \frac{1+r_{\mathrm{v}} / \epsilon}{1+r_{\mathrm{v}}+r_{1}}$

and by using the product rule,

$$
\begin{aligned}
\frac{\mathrm{d} \theta_{\rho}}{\mathrm{d} t}= & \frac{1+r_{\mathrm{v}} / \epsilon}{1+r_{\mathrm{v}}+r_{1}} \frac{\mathrm{d} \tilde{\theta}}{\mathrm{d} t}+\frac{\widetilde{\theta}}{1+r_{\mathrm{v}}+r_{1}} \frac{1}{\epsilon} \frac{\mathrm{d} r_{\mathrm{v}}}{\mathrm{d} t} \\
& -\tilde{\theta} \frac{1+r_{\mathrm{v}} / \epsilon}{\left(1+r_{\mathrm{v}}+r_{1}\right)^{2}}\left(\frac{\mathrm{d} r_{\mathrm{v}}}{\mathrm{d} t}+\frac{\mathrm{d} r_{1}}{\mathrm{~d} t}\right) \\
= & \frac{\rho_{\rho}}{\widetilde{\theta}} \frac{\mathrm{d} \tilde{\theta}}{\mathrm{d} t}+\theta_{\rho}\left(\frac{1}{\varepsilon+r_{\mathrm{v}}}-\frac{1}{1+r_{\mathrm{v}}+r_{1}}\right) \frac{\mathrm{d} r_{\mathrm{v}}}{\mathrm{d} t} \\
& -\frac{\theta_{\rho}}{1+r_{\mathrm{v}}+r_{1}} \frac{\mathrm{d} r_{1}}{\mathrm{~d} t} .
\end{aligned}
$$

Inserting Eqs. (A28), (A5) and (A6) in Eq. (A30):

$$
\begin{aligned}
\frac{\mathrm{d} \ln \theta_{\rho}}{\mathrm{d} t}= & \frac{\mathrm{d} \ln \tilde{\theta}}{\mathrm{d} t}+\left(\frac{1}{\varepsilon+r_{\mathrm{v}}}-\frac{1}{1+r_{\mathrm{v}}+r_{\mathrm{l}}}\right) \frac{\mathrm{d} r_{\mathrm{v}}}{\mathrm{d} t} \\
& -\frac{1}{1+r_{\mathrm{v}}+r_{1}} \frac{\mathrm{d} r_{1}}{\mathrm{~d} t} \\
= & -\frac{\ln \tilde{\pi}}{\rho_{\mathrm{d}}}\left(\frac{R_{\mathrm{v}}}{R_{\mathrm{m}}}-\frac{c_{\mathrm{pv}}}{c_{\mathrm{pml}}}\right) S_{\mathrm{v}} \\
& -\frac{1}{\rho_{\mathrm{d}}}\left(\frac{L_{\mathrm{v}}}{c_{\mathrm{pml}} T}+\ln \tilde{\pi}\left[\frac{R_{\mathrm{v}}}{R_{\mathrm{m}}}+\frac{c_{\mathrm{pl}}-c_{\mathrm{pv}}}{c_{\mathrm{pml}}}\right]\right) S_{\mathrm{ph}} \\
& -\frac{\ln \tilde{\pi}}{\rho_{\mathrm{d}}} \frac{c_{\mathrm{pl}}}{c_{\mathrm{pml}}} S_{\mathrm{fall}}-\frac{1}{\rho_{\mathrm{d}} c_{\mathrm{pml}} T} \frac{\partial}{\partial z}\left(\rho_{\mathrm{r}} W_{\mathrm{f}} e_{1}\right)-\frac{\rho_{\mathrm{r}} W_{\mathrm{f}} g}{\rho_{\mathrm{d}} c_{\mathrm{pml}} T} \\
& +\left(\frac{1}{\varepsilon+r_{\mathrm{v}}}-\frac{1}{1+r_{\mathrm{v}}+r_{\mathrm{l}}}\right) \frac{S_{\mathrm{v}}+S_{\mathrm{ph}}}{\rho_{\mathrm{d}}} \\
& +\frac{1}{1+r_{\mathrm{v}}+r_{1}} \frac{S_{\mathrm{ph}}+S_{\mathrm{fall}}}{\rho_{\mathrm{d}}} .
\end{aligned}
$$

With the relation

$$
\frac{1}{\varepsilon+r_{\mathrm{v}}}=\frac{1}{\frac{R_{\mathrm{d}}}{R_{\mathrm{v}}}+r_{\mathrm{v}}}=\frac{R_{\mathrm{v}}}{R_{\mathrm{d}}+r_{\mathrm{v}} R_{\mathrm{v}}}=\frac{R_{\mathrm{v}}}{R_{\mathrm{m}}},
$$

we get the density and potential temperature equation sorted by source terms:

$$
\begin{aligned}
\frac{\mathrm{d} \theta_{\rho}}{\mathrm{d} t} & =\frac{\theta_{\rho}}{\rho_{\mathrm{d}}}\left(\frac{R_{\mathrm{v}}}{R_{\mathrm{m}}}-\frac{\rho_{\mathrm{d}}}{\rho}-\ln \tilde{\pi}\left[\frac{R_{\mathrm{v}}}{R_{\mathrm{m}}}-\frac{c_{\mathrm{pv}}}{c_{\mathrm{pml}}}\right]\right) S_{\mathrm{v}} \\
& +\frac{\theta_{\rho}}{\rho_{\mathrm{d}}}\left(\frac{R_{\mathrm{v}}}{R_{\mathrm{m}}}-\ln \tilde{\pi}\left[\frac{R_{\mathrm{v}}}{R_{\mathrm{m}}}+\frac{c_{\mathrm{pl}}-c_{\mathrm{pv}}}{c_{\mathrm{pml}}}\right]-\frac{L_{\mathrm{v}}}{c_{\mathrm{pml}} T}\right) S_{\mathrm{ph}} \\
& +\frac{\theta_{\rho}}{\rho_{\mathrm{d}}}\left(\frac{\rho_{\mathrm{d}}}{\rho}-\frac{c_{\mathrm{pl}}}{c_{\mathrm{pml}}} \ln \tilde{\pi}\right) S_{\mathrm{fall}}-\frac{\theta_{\rho}}{\rho_{\mathrm{d}} c_{\mathrm{pml}} T} \frac{\partial}{\partial z}\left(\rho_{\mathrm{r}} W_{\mathrm{f}} e_{1}\right) \\
& -\frac{\theta_{\rho} \rho_{\mathrm{r}} W_{\mathrm{f}} g}{\rho_{\mathrm{d}} c_{\mathrm{pml}} T} .
\end{aligned}
$$




\section{Appendix B: Soil and land use parameters}

Varying ratios of silt, clay and sand significantly change the properties of soil and thus determine the heat and moisture fluxes of the surface. Accordingly, these different ratios are referred to specifically defined soil types. In Tables B1 and B2, parameters describing the physical properties of the appropriate soil type are listed. $W_{\mathrm{pv}}$ stands for the pore volume of the soil, $W_{\mathrm{fc}}$ is the field capacity describing a threshold value for runoff in the soil layers. $\kappa_{\text {sat }}$ and $\Psi_{\text {sat }}$ define the hydraulic conductivity and the matric potential at saturation, respectively (Eqs. 68,69). $\rho_{0} c_{0}$ is the heat capacity of dry soil as used in Eq. (71) and $b^{*}$ is a parameter for the soil porosity.

\section{Code availability and visualization}

The ASAM code is managed with Git, a distributed revision control and source code management (SCM) system. To get access to the source code and additional scripts for preand postprocessing, registration at the TROPOS Git hosting website https://gitorious.tropos.de/ is mandatory. Additional information can be found at the ASAM webpage (http: //asam.tropos.de).

As visualization tool, the free and open source software VisIt (https://wci.llnl.gov/codes/visit/) is used. VisIt can read over 120 scientific file formats and offers the opportunity to include own scripts, if necessary. It is available for Unix, Windows and Mac workstations.

Table B1. Soil parameters from Doms et al. (2011).

\begin{tabular}{lccccc}
\hline Soil type & $W_{\mathrm{pv}}\left(\mathrm{m}^{3} \mathrm{~m}^{-3}\right)$ & $W_{\mathrm{fc}}\left(\mathrm{m}^{3} \mathrm{~m}^{-3}\right)$ & $\kappa_{\mathrm{sat}}\left(\mathrm{m} \mathrm{s}^{-1}\right)$ & $\rho_{0} c_{0}\left(\mathrm{~W}\left(\mathrm{~m}^{3} \mathrm{~K}\right)^{-1}\right)$ & $b^{* \mathrm{a}}$ \\
\hline Sand & 0.364 & 0.196 & $4970 \times 10^{-8}$ & $1.28 \times 10^{6}$ & 3.5 \\
Sandy loam & 0.445 & 0.260 & $943 \times 10^{-8}$ & $1.35 \times 10^{6}$ & 4.8 \\
Loam & 0.455 & 0.340 & $531 \times 10^{-8}$ & $1.42 \times 10^{6}$ & 6.1 \\
Clay loam & 0.475 & 0.370 & $764 \times 10^{-8}$ & $1.50 \times 10^{6}$ & 8.6 \\
Clay & 0.507 & 0.463 & $1.7 \times 10^{-8}$ & $1.63 \times 10^{6}$ & 10.0 \\
Peat & 0.863 & 0.763 & $5.8 \times 10^{-8}$ & $0.58 \times 10^{6}$ & 9.0 \\
\hline
\end{tabular}

${ }^{\mathrm{a}}$ With $n=1 / b^{*}+1$, see Eqs. (68) and (69).

Table B2. Soil parameters as used in Pielke (1984) (adapted from McCumber, 1980).

\begin{tabular}{lccccc}
\hline Soil type & $W_{\mathrm{pv}}\left(\mathrm{m}^{3} \mathrm{~m}^{-3}\right)$ & $\Psi_{\mathrm{sat}}(\mathrm{m})$ & $\kappa_{\mathrm{sat}}\left(\mathrm{m} \mathrm{s}^{-1}\right)$ & $\rho_{0} c_{0}\left(\mathrm{~W}\left(\mathrm{~m}^{3} \mathrm{~K}\right)^{-1}\right)$ & $b^{* \mathrm{a}}$ \\
\hline Sand & 0.395 & -0.121 & $1760 \times 10^{-8}$ & $1.47 \times 10^{6}$ & 4.05 \\
Sandy Loam & 0.435 & -0.218 & $341 \times 10^{-8}$ & $1.34 \times 10^{6}$ & 4.90 \\
Loam & 0.451 & -0.478 & $70 \times 10^{-8}$ & $1.21 \times 10^{6}$ & 5.39 \\
Clay loam & 0.476 & -0.630 & $25 \times 10^{-8}$ & $1.23 \times 10^{6}$ & 8.52 \\
Clay & 0.482 & -0.405 & $13 \times 10^{-8}$ & $1.09 \times 10^{6}$ & 11.40 \\
Peat & 0.863 & -0.356 & $80 \times 10^{-8}$ & $0.84 \times 10^{6}$ & 7.75 \\
\hline
\end{tabular}

${ }^{\mathrm{a}}$ With $n=1 / b^{*}+1$, see Eqs. (68) and (69). 
Acknowledgements. This work is internally funded by TROPOS. The authors like to thank Luca Bonaventura and the anonymous reviewer for their constructive comments to improve the quality of the paper. We are also grateful to our technical employees Sabine Reutgen and Birgit Heinrich for developing and maintaining the grid generator as well as data maintenance and converting, respectively.

Edited by: H. Weller

\section{References}

Adcroft, A., Hill, C., and Marshall, J.: Representation of topography by shaved cells in a height coordinate ocean model, Mon. Weather Rev., 125, 2293-2315, 1997.

Berger, M. and Helzel, C.: A Simplified h-Box Method for Embedded Boundary Grids, Siam J. Sci. Comput., 34, A861-A888.

Bott, A.: Theoretical considerations on the mass and energy consistent treatment of precipitation in cloudy atmospheres, Atmos. Res., 89, 262-269, 2008.

Braun, F. J.: Mesoskalige Modellierung der Bodenhydrologie, Wiss. Berichte des Inst. f. Meteor. u. Klimat. d. Universität Karlsruhe, 30, 2002.

Bryan, G. H. and Fritsch, J. M.: A benchmark simulation for moist nonhydrostatic numerical models, Mon. Weather Rev., 130, 2917-2928, 2002.

Chen, F. and Dudhia, J.: Coupling an advanced land surfacehydrology model with the Penn State-NCAR MM5 modeling system. Part I: Model implementation and sensitivity, Mon. Weather Rev., 129, 569-585, 2001.

Cheng, Y. and Brutsaert, W.: Flux-profile relationships for wind speed and temperature in the stable atmospheric boundary layer, Bound.-Lay. Meteorol., 114, 519-53, 2005.

Deardorff, J. W.: Numerical investigation of neutral und unstable boundary layers, J. Atmos. Sci., 29, 91-115, 1972.

Doms, G., Förstner, J., Heise, E., Herzog, H.-J., Mironov, D., Raschendorfer, M., Reinhardt, T., Ritter, B., Schrodin, R., Schulz, J.-P., and Vogel, G.: A Description of the Nonhydrostatic Regional COSMO Model, Deutscher Wetterdienst, 2011.

Dongarra, J. J., Duff, I. S., Sorensen, D. C., and van der Vorst, H. A.: Numerical Linear Algebra for High-Performance Computers, Society for Industrial and Applied Mathematics, 1998.

Doyle, J. D., Gaberšek, S., Jiang, Q., Bernardet, L., Brown, J. M., Dörnbrack, A., Filaus, E., Grubišić, V., Knoth, O., Koch, S., Schmidli, J., Stiperski, I., Vosper, S. and Zhong, S.: An Intercomparison of T-REX Mountain-Wave Simulations and Implications for Mesoscale Predictability, Mon. Weather Rev., 139, 2811-2831, 2011.

Eisenstat, S.: Efficient implementation of a class of CG methods, SIAM J. Sci. Stat. Comput., 2, 1-4, 1981.

Emanuel, K. A.: Atmospheric Convection, Oxford University Press, 1994.

Engelmann, R., Ansmann, A., Horn, S., Seifert, P., Althausen, D., Tesche, M., Esselborn, M., Fruntke, J., Lieke, K., Freudenthaler, V., and Gross, S.: Doppler lidar studies of heat island effects on vertical mixing of aerosols during SAMUM-2, Tellus B, 63, 448-458, 2011.
Fairall, C. W., Bradley, E. F., Rogers, D. P., Edson, J. B., and Young, G. S.: Bulk parameterization of air-sea fluxes for Tropical Ocean Global Atmosphere Coupled-Ocean Atmosphere Response Experiment, J. Geophys. Res., 101, 3747-3764, 1996.

Good, B., Gadian, A., Lock, S. J., and Ross, A.: Performance of the cut-cell method of representing orography in idealized simulations, Atmos. Sci. Lett., 15, 44-49, 2014.

Hairer, E. and Wanner, G.: Solving Ordinary Differential Equations II, Springer, 1996

Hicken, J., Ham, F., Militzer, J., and Koksal, M.: A shift transformation for fully conservative methods: turbulence simulation on complex, unstructured grids, J. Comput. Phys., 208, 704-714, 2005.

Hinneburg, D. and Knoth, O.: Non-dissipative cloud transport in Eulerian grid models by the volume-of-fluid (VOF) method, Atmos. Environ., 39, 4321-4330, 2005.

Horn, S.: ASAMgpu V1.0 - a moist fully compressible atmospheric model using graphics processing units (GPUs), Geosci. Model Dev., 5, 345-353, doi:10.5194/gmd-5-345-2012, 2012.

Hundsdorfer, W., Koren, B., Loon, M. V., and Verwer, J. G.: A positive finite-difference advection scheme, J. Comput. Phys., 117 35-46, 1995.

Jebens, S., Knoth, O., and Weiner, R.: Partially implicit peer methods for the compressible Euler equations, J. Comput. Phys., 230, 4955-4974, 2011.

Jiménez, P. A., Dudhia, J., González-Rouco, J. F., Navarro, J., Montávez, J. P., and García-Bustamante, E.: A revised scheme for the WRF surface layer formulation, Mon. Weather Rev., 140, 898918, 2012.

John, V. and Rang, J.: Adaptive time step control for the incompressible Navier-Stokes equations, Comput. Methods Appl. Mech. Engrg., 199, 514-524, 2010.

Klein, R., Bates, K. R., and Nikiforakis, N.,: Well Balanced Compressible Cut-Cell Simulation of Atmospheric Flow, Phil. Trans. R. Soc. B, 367, 4559-4575, 2009.

Kleissl, J., Kumar, V., Meneveau, C., and Parlange, M. B.: Numerical study of dynamic Smagorinsky models in large-eddy simulation of the atmospheric boundary layer: validation in stable and unstable conditions, Water Resour. Res., 42, W06D10, doi:10.1029/2005WR004685, 2006.

König, M.: Large-eddy simulation modelling for urban Scale, Ph.D. thesis, University of Leipzig, 2013.

Knoth, O.: Compressible atmospheric modeling at all scales, in: Oberwolfach Reports 3, Workshop: Mathematical Theory and Modelling in Atmosphere-Ocean Science, 2374-2377, 2006.

Kong, L. C., Tunçel, L., and Xiu, N. H.: The Fischer-Burmeister complementarity function on Euclidean Jordan algebras, Pac. J. Optim., 6, 423-440, 2010.

Kunz, M. and Wassermann, S.: Sensitivity of flow dynamics and orographic precipitation to changing ambient conditions in idealised model simulations, Meteor. Z., 20, 199-215, doi:10.1127/0941-2948/2011/0221, 2011.

Lanser, D., Blom, J. G., and Verwer, J. G.: Time integration of the shallow water equations in spherical geometry, J. Comput. Phys., 171, 373-393, 2001.

Lilly, D. K.: On the numerical simulation of buoyant convection, Tellus, 14, 148-172, 1962. 
Lilly, D. K.: The representation of small scale turbulence in numerical simulation experiments, IBM Scientific Computing Symposium on environmental sciences, 195-210, 1967.

Lock, S.-J., Bitzer, H.-W., Coals, A., Gadian, A., and Mobbs, S.: Demonstration of a cut-cell representation of 3D orography for studies of atmospheric flows over very steep hills, Mon. Weather Rev., 140, 411-424, 2012.

McCumber, M. D.: A numerical simulation of the influence of heat and moisture fluxes upon mesoscale circulation, Ph.D. thesis, Dept. of Environmental Science, University of Virginia, Charlottesville, VA, 1980.

Ng, Y.-T., Chen, H., Min, C., and Gibou, F.: Guidelines for Poisson Solvers on Irregular Domains with Dirichlet Boundary Conditions Using the Ghost Fluid Method, J. Sci. Comput., 41, 300320, 2009

Noilhan, J. and Planton, S.: A simple parameterization of land surface processes for meteorological models, Mon. Weather Rev., 117, 536-549, 1989.

Pielke, R. A.: Mesoscale Meteorological Modeling, 2nd Edn., Academic Press, 1984.

Porté-Agel, F., Meneveau, C., and Parlange, M. B.: A scaledependent dynamic model for large-eddy simulation: application to a neutral atmospheric boundary layer, J. Fluid Mech., 415, 261-284, 2000

Satoh, M., Matsuno, T., Tomita, H., Miura, H., Nasuno, T., and Iga, S.: Nonhydrostatic icosahedral atmospheric model (NICAM) for global cloud resolving simulations, J. Comput. Phys., 227, 3486-3514, 2008.

Skamarock, W. C., Klemp, J. B., Duda, M. G., Fowler, L. D., Park, S.-H., and Ringler, T. D.: A multiscale nonhydrostatic atmospheric model using centroidal voronoi tesselations and c-grid staggering, Mon. Weather Rev., 140, 3090-3105, 2012.

Schaer, C., Leuenberger, D., Fuhrer, O., Lüthi, D., and Girard, C.: A new terrain-following vertical coordinate formulation for atmospheric prediction models, Mon. Weather Rev., 130, 2459-2480, 2002.

Scotti, A., Meneveau, C., and Lilly, D. K.: Generalized Smagorinsky model for anisotropic grids, Phys. Fluids A-Fluid, 5, 23062308, 1993.

Seifert, A. and Beheng, K. D.: A two-moment cloud microphysics parameterization for mixed-phase clouds, Meteorol. Atmos. Phys., 92, 45-66, 2006.

Skamarock, W. C., Klemp, J. B., Dudhia, J., Gill, D. O., Barker, M., Duda, K. G., Huang, X. Y., Wang, W., and Powers, J. G.: A description of the Advanced Research WRF Version 3, Tech. rep., National Center for Atmospheric Research, 2008.

Smagorinsky, J.: General circulation experiments with the primitive equations, Mon. Weather Rev., 164, 91-99, 1963.

Steppeler, J., Bitzer, H.-W., Minotte, M., and Bonaventura, L.: Nonhydrostatic atmospheric modeling using a z-coordinate representation, Mon. Weather Rev., 130, 2143-2149, 2002.
Steppeler, J., Bitzer, H.-W., Janjic, Z., Schättler, U., Prohl, P., Gjertsen, U., Torrisi, L., Parfinievicz, J., Avgoustoglou, E ., and Damrath, U.: Prediction of Clouds and Rain Using a z-Coordinate Nonhydrostatic Model, Mon. Weather Rev., 134, 3625-3643, 2006.

Steppeler, J., Park, S.-H. and Dobler, A.: Forecasts covering one month using a cut-cell model, Geosci. Model Dev., 6, 875-882, 2013.

Straka, J. M., Wilhelmson, R. B., Wicker, L. J., Anderson, J. R., and Droegemeier, K. K.: Numerical solutions of a non-linear density current: a benchmark solution and comparisons, Int. J. Numer. Meth. Fl., 17, 1-22, 1993.

Sweby, P. K.: High resolution schemes using flux limiters for hyperbolic conservation laws, SIAM J. Numer. Anal., 21, 995-1011, 1984.

Tripoli, G. J. and Smith, E. A.: Introducing Variable-Step Topography (VST) coordinates within dynamically constrained Nonhydrostatic Modeling System (NMS). Part 1: VST formulation within NMS host model framework, Dynam. Atmos. Oceans, 66, 28-57, 2014a.

Tripoli, G. J. and Smith, E. A.: Introducing Variable-Step Topography (VST) coordinates within dynamically constrained Nonhydrostatic Modeling System (NMS). Part 2: VST performance on orthodox obstacle flows, Dynam. Atmos. Oceans, 66, 10-27, 2014b.

Van Genuchten, M. T.: A closed-form equation for predicting the hydraulic conductivity of unsaturated soils, Soil Sci. Soc. Am. J., 44, 892-898, 1980.

Van Leer, B.: Upwind-difference methods for aerodynamic problems governed by the Euler equations, in: Lectures in Applied Mathematics, 327-336, 1994.

Walko, R. L. and Avissar, R.: The Ocean-Land-Atmosphere Model (OLAM). Part I: Shallow-water tests, Mon. Weather Rev., 136 , 4033-4044, 2008a.

Walko, R. L. and Avissar, R.: The Ocean-Land-Atmosphere Model (OLAM). Part II: Formulation and tests of the nonhydrostatic dynamic core, Mon. Weather Rev., 136, 4045-4062, 2008 b.

Wicker, L. J. and Skamarock, W. C.: A time-splitting scheme for the elastic equations incorporating second-order Runge-Kutta time differencing, Mon. Weather Rev., 126, 1992-1999, 1998.

Yamazaki, H. and Satomura, T.: Vertically combined shaved cell method in a $z$-coordinate nonhydrostatic atmospheric model, Atmos. Sci. Lett., 9, 171-175, 2008.

Yamazaki, H. and Satomura, T.: Nonhydrostatic Atmospheric Modeling Using a Combined Cartesian Grid, Mon. Weather Rev., 138, 3932-3945, 2010.

Yamazaki, H. and Satomura, T.: Non-hydrostatic atmospheric cut cell model on a block-structured mesh, Atmos. Sci. Lett., 13, 29_ 35, 2012. 